\title{
The canonical pencils on Horikawa surfaces
}

\author{
DENIS AUROUX
}

\begin{abstract}
We calculate the monodromies of the canonical Lefschetz pencils on a pair of homeomorphic Horikawa surfaces. We show in particular that the (pluri)canonical pencils on these surfaces have the same monodromy groups, and are related by a "partial twisting" operation.
\end{abstract}

57R17; 53D35, 14D05

\section{Introduction}

Horikawa surfaces are minimal complex surfaces of general type which realize the equality case in Noether's inequality $c_{1}^{2} \geq 2 p_{g}-4$. While their classification as complex surfaces has been completed a long time ago [16], the topology of these surfaces viewed as smooth 4-manifolds, or as symplectic 4-manifolds, remains mysterious. In this paper we consider two specific Horikawa surfaces:

Definition 1.1 Denote by $X_{1}$ a double cover of $\mathbb{C P}^{1} \times \mathbb{C P}^{1}$ branched along a smooth algebraic curve $C_{1}$ of bidegree $(6,12)$. Denote by $X_{2}$ a double cover of the Hirzebruch surface $\mathbb{F}_{6}=\mathbb{P}\left(\mathcal{O}_{\mathbb{P} 1} \oplus \mathcal{O}_{\mathbb{P}^{1}}(6)\right)$ branched along $\Delta_{\infty} \cup C_{2}$, where $\Delta_{\infty}$ is the exceptional section of $\mathbb{F}_{6}\left(\Delta_{\infty} \cdot \Delta_{\infty}=-6\right)$, and $C_{2}$ is a smooth algebraic curve in the linear system $\left|5 \Delta_{0}\right|$ (where $\Delta_{0}$ is the zero section, satisfying $\Delta_{0} \cdot \Delta_{0}=+6$ ).

(The actual choices of $C_{1}$ and $C_{2}$ are irrelevant from the point of view of symplectic topology, hence we do not specify them here).

The complex surfaces $X_{1}$ and $X_{2}$ are simply-connected, non-spin, and have the same Euler characteristic $e\left(X_{1}\right)=e\left(X_{2}\right)=116$ and signature $\sigma\left(X_{1}\right)=\sigma\left(X_{2}\right)=$ -72 ; hence by a classical result of Freedman they are homeomorphic. Moreover, the homeomorphism between them can be chosen so that the canonical classes $K_{X_{1}}$ and $K_{X_{2}}$ are the image of each other under the induced map on cohomology. However, $X_{1}$ and $X_{2}$ are not deformation equivalent as complex surfaces [16]. The question of whether $X_{1}$ and $X_{2}$ are diffeomorphic is open to this date; although the expected answer is negative, a result of Friedman and Morgan [10] shows that $X_{1}$ and $X_{2}$ cannot be distinguished using Seiberg-Witten theory. 
Since the canonical classes $K_{X_{1}}, K_{X_{2}}$ are ample, one can equip $X_{1}$ and $X_{2}$ with Kähler forms such that $\left[\omega_{i}\right]=c_{1}\left(K_{X_{i}}\right)$ and view them as symplectic 4-manifolds. The question of whether $\left(X_{1}, \omega_{1}\right)$ and $\left(X_{2}, \omega_{2}\right)$ are symplectomorphic is again open; following a strategy proposed by Donaldson [7], one can try to approach this problem from the perspective of Lefschetz pencils.

Namely, considering a generic pencil of curves in the linear system $\left|K_{X_{i}}\right|$ and blowing up its 16 base points, we obtain a fibration $\widehat{f}_{i}: \hat{X}_{i} \rightarrow \mathbb{C P}^{1}$. The generic fiber of $\hat{f}_{i}$ is a smooth curve of genus 17, and the singular fibers are nodal (there are 196 nodes in total); moreover, the exceptional divisors of the blowups determine 16 distinguished sections of $\hat{f}_{i}$. It is well-known (see Section 2.1) that the monodromy of such a fibration can be described by an ordered tuple of Dehn twists in the mapping class group Map ${ }_{17,16}$ of a genus 17 surface with 16 boundary components (up to Hurwitz equivalence and global conjugation).

Theorem 1.2 The canonical pencils on $X_{1}$ and $X_{2}$ are related by a "partial twisting" operation, ie, there exist Dehn twists $\phi, t_{1}, \ldots, t_{196} \in$ Map $_{17,16}$ such that the monodromy of $\hat{f}_{1}$ can be expressed by the tuple $\left(\phi t_{1} \phi^{-1}, \ldots, \phi t_{64} \phi^{-1}, t_{65}, \ldots, t_{196}\right)$, and the monodromy of $\hat{f}_{2}$ can be expressed by the tuple $\left(t_{1}, \ldots, t_{64}, t_{65}, \ldots, t_{196}\right)$.

There is a geometric reason for this property of the canonical pencils: the Horikawa surfaces $X_{1}$ and $X_{2}$ can be obtained from each other by a Luttinger surgery operation (cf Theorem 4.3).

In fact, we determine the monodromies of $\widehat{f}_{1}$ and $\widehat{f}_{2}$ explicitly (the formulas are given in Theorems 3.2 and 4.4); as a consequence, we also get:

Theorem 1.3 The monodromy groups of $\widehat{f}_{1}$ and $\widehat{f}_{2}$, ie, the subgroups of Map 17,16 $_{10}$ generated by the Dehn twists in their monodromy, are isomorphic to each other.

All these results suggest that (somewhat unsurprisingly) the pencils $\hat{f}_{1}$ and $\hat{f}_{2}$ are very similar to each other, and difficult to tell apart. This is in sharp contrast with the well-known genus 2 fibrations carried by $X_{1}$ and $X_{2}$, whose monodromies are easily distinguished (see Section 2.3).

By the work of Gompf $[13 ; 12]$, if the tuples of Dehn twists describing $\widehat{f}_{1}$ and $\widehat{f}_{2}$ are Hurwitz and conjugation equivalent, then $\left(X_{1}, \omega_{1}\right)$ and $\left(X_{2}, \omega_{2}\right)$ are symplectomorphic. The converse is not necessarily true. However, by Donaldson's asymptotic uniqueness result for symplectic Lefschetz pencils [8], if $\left(X_{1}, \omega_{1}\right)$ and $\left(X_{2}, \omega_{2}\right)$ are symplectomorphic then there exists an integer $k_{0}$ such that the pluricanonical Lefschetz pencils on $X_{1}$ and $X_{2}$ (ie, generic pencils of curves in the linear systems $\left|k K_{X_{i}}\right|$ ) 
have equivalent monodromies for all $k \geq k_{0}$. Hence, in order to prove that $\left(X_{1}, \omega_{1}\right)$ and $\left(X_{2}, \omega_{2}\right)$ are not symplectomorphic, one needs to compare not just the canonical pencils of $X_{1}$ and $X_{2}$, but also a sequence of pluricanonical pencils.

"Degree doubling" arguments [3;26] (see also Section 7) imply that the monodromies of the pluricanonical pencils (for the linear systems $\left|2^{m} K_{X_{i}}\right|$ ) are determined by those of the canonical pencils in an explicit and "universal" manner. In particular, it is expected that an invariant that distinguishes the canonical pencils should also be able to distinguish the pluricanonical pencils (and hence prove that $X_{1}$ and $X_{2}$ are not symplectomorphic). While no such invariant is known to this date, it is still worth mentioning the following consequence of Theorems 1.2 and 1.3:

Theorem 1.4 For all $m \geq 0$, generic pencils of curves in the linear systems $\left|2^{m} K_{X_{i}}\right|$ on $X_{1}$ and $X_{2}$ are related to each other by "partial twisting" operations, and their monodromy subgroups are isomorphic.

The rest of this paper is organized as follows: in Section 2 we review some background material on Lefschetz fibrations and their monodromy (Section 2.1), lifting homomorphisms (Section 2.2), Horikawa surfaces (Section 2.3), and Luttinger surgery (Section 2.4). Section 3 is devoted to the calculation of the monodromy of the canonical pencil on $X_{2}$. In Section 4 we show that $X_{1}$ and $X_{2}$ are related by Luttinger surgery, and which allows us to determine the monodromy of a certain symplectic Lefschetz pencil on $X_{1}$; using the theory of pseudo-holomorphic curves, we prove in Section 5 that this Lefschetz pencil is isomorphic to the canonical pencil of $X_{1}$. This allows us to complete the proof of Theorem 1.2, while Theorem 1.3 is proved in Section 6. The paper ends with considerations about degree doubling and pluricanonical pencils in Section 7, and Lagrangian spheres and matching paths in Section 8.

While we are still a long way from proving that $X_{1}$ and $X_{2}$ are not symplectomorphic, the explicit calculation of the monodromies of their canonical pencils sheds some light on the situation; we hope that it will lead to further advances on this problem, and give some insight about what kind of invariants one might consider in order to distinguish homeomorphic surfaces of general type.

Acknowledgements This work was partially supported by NSF grants DMS-0244844 and DMS-0600148 and an A P Sloan research fellowship. The author wishes to thank the Department of Mathematics at UC Berkeley for its hospitality during part of the preparation of this work.

Geometry $\&$ Topology, Volume 10 (2006) 


\section{Preliminaries}

\subsection{Lefschetz fibrations and symplectic 4-manifolds}

Definition 2.1 A Lefschetz fibration on an oriented compact smooth 4-manifold $M$ is a smooth map $f: M \rightarrow S^{2}$ which is a submersion everywhere except at finitely many non-degenerate critical points $p_{1}, \ldots, p_{r}$, near which $f$ identifies in local orientation-preserving complex coordinates with the model map $\left(z_{1}, z_{2}\right) \mapsto z_{1}^{2}+z_{2}^{2}$.

The fibers of a Lefschetz fibration $f$ are compact oriented surfaces, smooth except for finitely many of them. The fiber through $p_{i}$ presents a transverse double point, or node, at $p_{i}$. Without loss of generality, we can assume after perturbing $f$ slightly that the critical values $q_{i}=f\left(p_{i}\right)$ are all distinct. Fix a reference point $q_{*}$ in $S^{2} \backslash \operatorname{crit}(f)$, and let $\Sigma=f^{-1}\left(q_{*}\right)$ be the corresponding fiber. Then we can consider the monodromy homomorphism

$$
\psi: \pi_{1}\left(S^{2} \backslash \operatorname{crit}(f), q_{*}\right) \rightarrow \operatorname{Map}(\Sigma),
$$

where $\operatorname{Map}(\Sigma)=\pi_{0} \operatorname{Diff}^{+}(\Sigma)$ is the mapping class group of $\Sigma$. The image $\psi(\gamma)$ of a loop $\gamma \subset S^{2} \backslash \operatorname{crit}(f)$ is the isotopy class of the diffeomorphism of $\Sigma$ induced by parallel transport (with respect to an arbitrary horizontal distribution) along the loop $\gamma$.

The singular fibers of $f$ are obtained from the nearby smooth fibers by collapsing a simple closed loop, called the vanishing cycle. The monodromy of a Lefschetz fibration around a singular fiber is the positive Dehn twist along the corresponding vanishing cycle. Choose an ordered collection $\eta_{1}, \ldots, \eta_{r}$ of arcs joining $q_{*}$ to the various critical values of $f$, and thicken them to obtain closed loops $\gamma_{1}, \ldots, \gamma_{r}$ based at $q_{*}$ in $S^{2} \backslash \operatorname{crit}(f)$, such that each $\gamma_{i}$ encircles exactly one of the critical values of $f$, and $\pi_{1}\left(S^{2} \backslash \operatorname{crit}(f), q_{*}\right)=\left\langle\gamma_{1}, \ldots, \gamma_{r} \mid \prod \gamma_{i}=1\right\rangle$. Then the monodromy of $f$ along each $\gamma_{i}$ is a positive Dehn twist $t_{i}$ along an embedded loop $\delta_{i} \subset \Sigma$, obtained by parallel transport along $\eta_{i}$ of the vanishing cycle at the critical point $p_{i}$, and in $\operatorname{Map}(\Sigma)$ we have the relation $t_{1} \ldots t_{r}=$ Id.

Hence, to every Lefschetz fibration we can associate a factorization of the identity element as a product of positive Dehn twists in the mapping class group of the fiber, ie, an ordered tuple of Dehn twists whose product is equal to Id; we will often use the multiplicative notation, with the understanding that what is important is not the product of the factors but rather the factors themselves.

Given the collection of Dehn twists $t_{1}, \ldots, t_{r}$ we can reconstruct the Lefschetz fibration $f$ above a large disc $\mathrm{D}$ containing all the critical values, by starting from $\Sigma \times D^{2}$ and adding handles as specified by the vanishing cycles [17]. To recover the 4-manifold $M$ we need to glue $f^{-1}(\mathrm{D})$ and the trivial fibration $f^{-1}\left(S^{2} \backslash \mathrm{D}\right)=\Sigma \times D^{2}$ along 
their common boundary, in a manner compatible with the fibration structures. In general this gluing involves the choice of an element in $\pi_{1} \operatorname{Diff}^{+}(\Sigma)$; however the diffeomorphism group is simply connected if the genus of $\Sigma$ is at least 2, and in that case the factorization $t_{1} \ldots t_{r}=$ Id determines the Lefschetz fibration $f: M \rightarrow S^{2}$ completely (up to isotopy).

The monodromy factorization $t_{1} \ldots t_{r}=$ Id depends not only on the topology of $f$, but also on the choice of an ordered collection $\gamma_{1}, \ldots, \gamma_{r}$ of generators of $\pi_{1}\left(S^{2} \backslash\right.$ $\left.\operatorname{crit}(f), q_{*}\right)$; the braid group $B_{r}$ acts transitively on the set of all such ordered collections, by Hurwitz moves. The equivalence relation induced by this action on the set of mapping class group factorizations is generated by

$$
\left(t_{1}, \ldots, t_{i}, t_{i+1}, \ldots, t_{r}\right) \sim\left(t_{1}, \ldots, t_{i} t_{i+1} t_{i}^{-1}, t_{i}, \ldots, t_{r}\right) \quad \forall 1 \leq i<r,
$$

and is called Hurwitz equivalence. Additionally, in order to remove the dependence on the choice of the reference fiber $\Sigma$, we should view the Dehn twists $t_{i}$ as elements of the mapping class group Map $g$ of an abstract surface of genus $g=g(\Sigma)$. This requires the choice of an identification diffeomorphism, and introduces another equivalence relation on the set of mapping class group factorizations: global conjugation,

$$
\left(t_{1}, \ldots, t_{r}\right) \sim\left(\phi t_{1} \phi^{-1}, \ldots, \phi t_{r} \phi^{-1}\right) \quad \forall \phi \in \operatorname{Map}_{g} .
$$

Proposition 2.2 For $g \geq 2$, there is a one to one correspondence between (a) factorizations of Id as a product of positive Dehn twists in $\mathrm{Map}_{g}$, up to Hurwitz equivalence and global conjugation, and $(b)$ genus $g$ Lefschetz fibrations over $S^{2}$, up to isomorphism.

It is a classical result of Thurston that, if $M$ is an oriented surface bundle over an oriented surface, then $M$ is a symplectic 4-manifold, at least provided that the homology class of the fiber is nonzero in $H_{2}(M, \mathbb{R})$. As shown by Gompf, the argument extends to the case of Lefschetz fibrations [13, Theorem 10.2.18]:

Theorem 2.3 (Gompf) Let $f: M \rightarrow S^{2}$ be a Lefschetz fibration, and assume that the fiber represents a nonzero class in $H_{2}(M, \mathbb{R})$. Then $M$ admits a symplectic structure for which the fibers of $f$ are symplectic submanifolds; this symplectic structure is unique up to deformation.

Lefschetz fibrations arise naturally in algebraic geometry: if $X$ is a complex surface, and $L \rightarrow X$ is a sufficiently ample line bundle, then the ratio between two suitably chosen sections $s_{0}, s_{1} \in H^{0}(L)$ determines a Lefschetz pencil, ie, a map $f=\left(s_{0} / s_{1}\right): X \backslash B \rightarrow$ $\mathbb{C P} P^{1}$, defined on the complement of the finite set $B=\left\{s_{0}=s_{1}=0\right\}$ (the base points), with isolated nondegenerate critical points in $X \backslash B$. 
More generally, Donaldson has shown that this construction extends to the symplectic setting [8]:

Theorem 2.4 (Donaldson) Let $(X, \omega)$ be a compact symplectic 4-manifold with $[\omega] \in H^{2}(X, \mathbb{Z})$. Then $X$ carries a symplectic Lefschetz pencil, ie, there exist a finite set $B \subset X$ and a map $f: X \backslash B \rightarrow \mathbb{C P}^{1}=S^{2}$ such that $f$ is modelled on $\left(z_{1}, z_{2}\right) \mapsto\left(z_{1}: z_{2}\right)$ near each point of $B$, and $f$ is a Lefschetz fibration with symplectic fibers outside of $B$.

More precisely, given a compact symplectic 4-manifold $(X, \omega)$ such that $[\omega] \in$ $H^{2}(X, \mathbb{Z})$, and given a complex line bundle $L \rightarrow X$ with $c_{1}(L)=[\omega]$, one can construct symplectic Lefschetz pencils from suitably chosen pairs of sections $s_{0}, s_{1} \in C^{\infty}\left(L^{\otimes k}\right)$ for all sufficiently large values of the integer $k$. Moreover, Donaldson has shown that for all sufficiently large values $k$, there is a distinguished connected component of the space of symplectic Lefschetz pencils obtained from pairs of sections of $L^{\otimes k}$; in the Kähler case, this component contains pencils defined from pairs of generic holomorphic sections of $L^{\otimes k}$ [8]. Hence, if two complex projective surfaces are symplectomorphic, then generic pencils of curves in the linear systems considered by Donaldson are mutually isomorphic (as symplectic Lefschetz pencils) whenever the integer $k$ is sufficiently large.

Given a symplectic Lefschez pencil $f: X \backslash B \rightarrow S^{2}$, the manifold $\hat{X}$ obtained from $X$ by blowing up the points of $B$ admits a Lefschetz fibration $\hat{f}: \hat{X} \rightarrow S^{2}$ with symplectic fibers, and can be described by its monodromy as discussed above.

Moreover, the fibration $\hat{f}$ has $n=|B|$ distinguished sections $e_{1}, \ldots, e_{n}$, corresponding to the exceptional divisors of the blowups. Therefore, each fiber of $\hat{f}$ comes equipped with $n$ marked points, and the monodromy of $\hat{f}$ lifts to the mapping class group of a genus $g$ surface with $n$ marked points.

The normal bundles of the sections $e_{i}$ are not trivial, but it is possible to trivialize them over the preimage of a large disc $D$ containing all the chosen generators of $\pi_{1}\left(S^{2} \backslash \operatorname{crit}(\widehat{f})\right)$. Deleting a small tubular neighborhood of each exceptional section, we can now view the monodromy of $\hat{f}$ as a morphism

$$
\widehat{\psi}: \pi_{1}(\mathrm{D} \backslash \operatorname{crit}(\widehat{f})) \rightarrow \operatorname{Map}_{g, n},
$$

where $\operatorname{Map}_{g, n}$ is the mapping class group of a genus $g$ surface with $n$ boundary components (ie, $\left.\pi_{0} \operatorname{Diff}^{+}(\Sigma, \partial \Sigma)\right)$.

The product of the Dehn twists $t_{i}=\widehat{\psi}\left(\gamma_{i}\right)$ is no longer the identity element in $\operatorname{Map}_{g, n}$. Instead, since $\prod \gamma_{i}$ is homotopic to the boundary of the disc $\mathrm{D}$, and since the normal 
bundle to $e_{i}$ has degree -1 , we have $\prod t_{i}=T_{\partial}$, where $T_{\partial} \in \operatorname{Map}_{g, n}$ is the boundary twist, ie, the product of the positive Dehn twists along $n$ loops parallel to the boundary components.

With this understood, the previous discussion carries over, and under the assumption $2-2 g-n<0$ there is a one to one correspondence between factorizations of the boundary twist $T_{\partial}$ as a product of positive Dehn twists in $\operatorname{Map}_{g, n}$, up to Hurwitz equivalence and global conjugation, and genus $g$ Lefschetz fibrations over $S^{2}$ equipped with $n$ distinguished sections of square -1 , up to isomorphism.

Moreover, Theorem 2.3 admits a strengthening in this context: for symplectic Lefschetz pencils whose fibers are Poincaré dual to a symplectic form, the monodromy data determines the symplectic structure up to isotopy (ie, symplectomorphism), rather than just up to deformation [12]. Combining this with the discussion after Theorem 2.4, we conclude:

Corollary 2.5 (Donaldson, Gompf) The following three properties are equivalent:

(i) the Horikawa surfaces $X_{1}$ and $X_{2}$ equipped with their canonical Kähler forms are symplectomorphic;

(ii) there exists an integer $k \geq 1$ such that generic pencils of curves in the linear systems $\left|k K_{X_{i}}\right|$ have equivalent monodromy factorizations;

(iii) there exists an integer $k_{0}$ such that, for all $k \geq k_{0}$, generic pencils of curves in the linear systems $\left|k K_{X_{i}}\right|$ have equivalent monodromy factorizations.

\subsection{Double covers and lifting homomorphisms}

Let $X$ and $Y$ be smooth complex surfaces, such that there exists a 2:1 covering map $\pi: X \rightarrow Y$, branched along a smooth curve $C \subset Y$. Assume that we have a Lefschetz pencil $f: Y \backslash B \rightarrow \mathbb{C P}^{1}$ (we also allow $f$ to be a fibration, ie, $B$ may be empty), and that the branch curve $C$ satisfies the following properties:

(i) $C$ does not pass through the base points or the critical points of $f$;

(ii) $C$ is everywhere transverse to the fibers of $f$, except at isolated points $p_{1}, \ldots, p_{s}$ where $C$ is nondegenerately tangent to the fiber of $f$ (ie, at $p_{i}$ the multiplicity of the intersection between $C$ and the fiber of $f$ is 2);

(iii) for simplicity we also assume that the points $p_{i}$ lie in distinct smooth fibers of the pencil $f$.

Then $\tilde{f}=f \circ \pi: X \backslash \widetilde{B} \rightarrow \mathbb{C} \mathbb{P}^{1}$ is also a Lefschetz pencil, with base points $\widetilde{B}=\pi^{-1}(B)$. 
Remark 2.6 The discussion extends without modification to the situation where $\pi: X \rightarrow Y$ is a branched covering of symplectic 4 -manifolds with a smooth symplectic branch curve, and $f$ is a symplectic Lefschetz pencil.

Denote by $\Sigma$ the generic fiber of $f$, with a neighborhood of the base points removed (so $\Sigma$ is a compact surface with $n=|B|$ boundary components, and the monodromy of $f$ takes values in the mapping class group of $\Sigma$ ). The generic fiber of $\tilde{f}$ is a double cover of $\Sigma$ branched at $d=[C] \cdot[\Sigma]$ points, which we denote by $\widetilde{\Sigma}$. Abusing notation, we denote the restriction of the double cover to the fiber by the same letter: $\pi: \widetilde{\Sigma} \rightarrow \Sigma$.

It is a classical fact that the double covering $\pi$ determines a lifting homomorphism $L$ from (a subgroup of) the braid group $B_{d}(\Sigma)$ (ie, the fundamental group of the space $\mathcal{C}_{d}(\Sigma)$ of unordered configurations of $d$ distinct points in the interior of $\Sigma$ ) to the mapping class group of $\tilde{\Sigma}$. In the case where $\Sigma$ has genus 0 , which is the only one we will be considering, one way to describe the lifting homomorphism is to consider the universal family $\mathcal{X} \rightarrow \mathcal{C}_{d}(\Sigma)$ whose fiber above a configuration $\left\{x_{1}, \ldots, x_{d}\right\} \subset \Sigma$ is the double cover of $\Sigma$ branched at $x_{1}, \ldots, x_{d}$ (with trivial monodromy along each component of $\partial \Sigma$ ). (When the genus of $\Sigma$ is nonzero, this universal family is only defined over a finite covering of $\mathcal{C}_{d}(\Sigma)$ ). The lifting homomorphism is simply the monodromy of the fibration $\mathcal{X} \rightarrow \mathcal{C}_{d}(\Sigma)$; however not every element of $B_{d}(\Sigma)$ is liftable, because some braids lift to diffeomorphisms of $\widetilde{\Sigma}$ which exchange the two lifts of some boundary components of $\Sigma$ instead of fixing $\partial \widetilde{\Sigma}$ pointwise.

Given any arc $\eta$ joining two points $x_{i}^{0}$ and $x_{j}^{0}$ of the reference configuration $\left\{x_{1}^{0}, \ldots, x_{d}^{0}\right\}=\Sigma \cap C \subset \Sigma$ inside $\Sigma \backslash\left\{x_{1}^{0}, \ldots, x_{d}^{0}\right\}$, we can consider the half-twist along $\eta$, which is the braid exchanging the two points $x_{i}^{0}$ and $x_{j}^{0}$ by a counterclockwise 180 degree rotation inside a small tubular neighborhood of $\eta$. The preimage $\pi^{-1}(\eta)$ is a simple closed curve in $\widetilde{\Sigma}$, and it is a classical observation that the half-twist along $\eta$ lifts to the Dehn twist along $\pi^{-1}(\eta)$.

We claim that the monodromy of the Lefschetz fibration $\tilde{f}$ is completely determined by the monodromy of $f$ and by the braid monodromy of the branch curve $C$. Indeed, the singular fibers of $\widetilde{f}$ are of two types:

(a) preimages by $\pi$ of the singular fibers of $f$;

(b) preimages by $\pi$ of smooth fibers of $f$ which are tangent to $C$.

Denote by $q_{1}, \ldots, q_{r}$ the critical points of $f$, which we assume to lie in distinct fibers, and choose an ordered collection of generating loops for $\pi_{1}(\mathrm{D} \backslash \operatorname{crit}(\tilde{f}))$, where $\mathrm{D}$ is a large disc containing all the points of $\operatorname{crit}(\tilde{f})=\left\{f\left(q_{1}\right), \ldots, f\left(q_{r}\right), f\left(p_{1}\right), \ldots, f\left(p_{s}\right)\right\}$. 
This allows us to define the monodromy of $\tilde{f}$ around its various critical values as in Section 2.1.

First consider a singular fiber of $f$, containing a critical point $q_{j}$. The corresponding fiber of $\tilde{f}$ possesses two nodal singularities (the two preimages of $q_{j}$ ), and we claim that the corresponding vanishing cycles are the two lifts by $\pi$ of the vanishing cycle at $q_{j}$.

Indeed, by assumption $q_{j} \notin C$, and since $C$ is transverse to the fibers of $f$ in the considered region, we can locally choose the parallel transport maps between the various fibers of $f$ in a manner such that the intersection points with $C$ are preserved. Therefore, the vanishing cycle of $f$ associated to the critical point $q_{j}$ and to the chosen generator of $\pi_{1}(\mathrm{D} \backslash \operatorname{crit}(\tilde{f}))$ can be naturally represented by a simple closed curve $\delta_{j} \subset \Sigma \backslash\left\{x_{1}^{0}, \ldots, x_{d}^{0}\right\}$. The preimage $\pi^{-1}\left(\delta_{j}\right)$ consists of two disjoint simple closed curves $\delta_{j}^{\prime}$ and $\delta_{j}^{\prime \prime}$, which are precisely the two vanishing cycles of $\widetilde{f}$. The monodromy of $f$ along the chosen loop around $q_{j}$ is the Dehn twist along $\delta_{j}$, and the monodromy of $\tilde{f}$ along the same loop is the product of the Dehn twists along $\delta_{j}^{\prime}$ and $\delta_{j}^{\prime \prime}$.

Remark 2.7 By construction, the critical values of $\tilde{f}$ are not distinct, since critical points of $f$ lift to pairs of critical points in the same fiber. However, when the chosen linear system is sufficiently ample, the critical values can be made distinct by considering a small generic perturbation of $\tilde{f}$; if the perturbation is chosen sufficiently small then the vanishing cycles are not affected. More generally, even when such a perturbation does not exist in the algebraic setting (as is the case for canonical pencils on Horikawa surfaces), it can still be carried out among symplectic Lefschetz pencils. Hence, when viewing Horikawa surfaces as symplectic 4-manifolds it is natural (and desirable) to consider the individual Dehn twists arising in the monodromy, even though the critical values of the pencils are not pairwise distinct.

We now consider the fiber of $f$ through the point $p_{j}$. Denote by $\gamma_{j}$ the loop around $f\left(p_{j}\right)$ chosen as part of our fixed collection of generators of $\pi_{1}(\mathrm{D} \backslash \operatorname{crit}(\tilde{f}))$, and by $\mathrm{D}_{j}$ the disc bounded by $\delta_{j}$. Since $f$ has no critical values in $\mathrm{D}_{j}$, we can identify $f^{-1}\left(\mathrm{D}_{j}\right)$ with $\mathrm{D}_{j} \times \Sigma$. The branch curve $C$ intersects the fiber of $f$ above any point $z \in \mathrm{D}_{j} \backslash\left\{f\left(p_{j}\right)\right\}$ transversely in $d$ distinct points, which determines an element $\sigma(z)$ of the configuration space $\mathcal{C}_{d}(\Sigma)$ (using the trivialization of $f$ ). The manner in which two of the points in these configurations converge to each other (while remaining distinct from the others) as $z$ approaches $f\left(p_{j}\right)$ is encoded by a vanishing arc $\eta_{j} \subset \Sigma \backslash\left\{x_{1}^{0}, \ldots, x_{d}^{0}\right\}$, with end points in $\left\{x_{1}^{0}, \ldots, x_{d}^{0}\right\}$ (recall that we denote by $\left\{x_{1}^{0}, \ldots, x_{d}^{0}\right\} \subset \Sigma$ the configuration above the base point). Local models for $C$ 
and $f$ near $p_{j}$ are given by the plane curve $\left\{y^{2}=x\right\} \subset \mathbb{C}^{2}$ and the projection to the first coordinate. Therefore, as one moves around $f\left(p_{j}\right)$, the two end points of the vanishing arc are exchanged by a counterclockwise half-twist. It follows that the braid monodromy of $C$ along $\gamma_{j}$, ie, the element of $B_{d}(\Sigma)$ determined by the configurations $\left\{\sigma(z), z \in \gamma_{j}\right\}$, is precisely the half-twist along the vanishing arc $\eta_{j}$. By construction, the monodromy of $\tilde{f}$ along $\gamma_{j}$ is the image of this half-twist under the lifting homomorphism, ie, the Dehn twist along $\pi^{-1}\left(\eta_{j}\right)$.

In conclusion, we have proved:

Proposition 2.8 The vanishing cycles of $\tilde{f}$ are exactly the preimages by $\pi: \widetilde{\Sigma} \rightarrow \Sigma$ of $(a)$ the vanishing cycles of $f$, and $(b)$ the vanishing arcs of the branch curve $C$.

\subsection{Horikawa surfaces as genus 2 fibrations}

The composition of the covering map $\pi_{1}: X_{1} \rightarrow \mathbb{C P}^{1} \times \mathbb{C P}^{1}$ with the projection to the first factor defines a genus 2 fibration $\varphi_{1}: X_{1} \rightarrow \mathbb{C P}^{1}$. A generic choice of the branch curve $C_{1} \subset \mathbb{C P}^{1} \times \mathbb{C P}^{1}$ ensures that every fiber of the projection to the first factor is tangent to $C_{1}$ in at most one point, and that every such tangency is nondegenerate. We can then derive the monodromy of the Lefschetz fibration $\varphi_{1}$ from the braid monodromy of the curve $C_{1}$, as in Section 2.2. It is easy to check (see eg [1, Section 3]) that the braid monodromy of $C_{1}$ corresponds to the factorization

$$
\left(\sigma_{1} \cdot \sigma_{2} \cdot \sigma_{3} \cdot \sigma_{4} \cdot \sigma_{5} \cdot \sigma_{5} \cdot \sigma_{4} \cdot \sigma_{3} \cdot \sigma_{2} \cdot \sigma_{1}\right)^{12}=1
$$

in the spherical braid group $B_{6}\left(S^{2}\right)$, where $\sigma_{1}, \ldots, \sigma_{5}$ are the standard Artin generators (half-twists exchanging two consecutive points).

Similarly, the composition of the covering map $\pi_{2}: X_{2} \rightarrow \mathbb{F}_{6}$ with the projection pr: $\mathbb{F}_{6} \rightarrow \mathbb{C P}^{1}$ defines a genus 2 fibration $\varphi_{2}: X_{2} \rightarrow \mathbb{C P}^{1}$; the braid monodromy of the branch curve $\Delta_{\infty} \cup C_{2}$ can again be expressed in terms of a factorization in $B_{6}\left(S^{2}\right)$, namely

$$
\left(\sigma_{1} \cdot \sigma_{2} \cdot \sigma_{3} \cdot \sigma_{4}\right)^{30}=1 .
$$

Using the fact that the half-twists $\sigma_{1}, \ldots, \sigma_{5}$ lift to the Dehn twists $\tau_{1}, \ldots, \tau_{5} \in \mathrm{Map}_{2}$ represented in Figure 1 (the standard generators of $\mathrm{Map}_{2}$ ), we obtain formulas for the monodromies of the Lefschetz fibrations $\varphi_{1}$ and $\varphi_{2}$. For another derivation of these formulas, see the work of Fuller [11]; see also [23, Section 4] for related considerations.

Proposition 2.9 (Fuller) $X_{1}$ and $X_{2}$ admit genus 2 Lefschetz fibrations with 120 singular fibers; the corresponding monodromy factorizations in $\mathrm{Map}_{2}$ are $\left(\tau_{1} \cdot \tau_{2} \cdot \tau_{3}\right.$. $\left.\tau_{4} \cdot \tau_{5} \cdot \tau_{5} \cdot \tau_{4} \cdot \tau_{3} \cdot \tau_{2} \cdot \tau_{1}\right)^{12}=1$ and $\left(\tau_{1} \cdot \tau_{2} \cdot \tau_{3} \cdot \tau_{4}\right)^{30}=1$, respectively. 


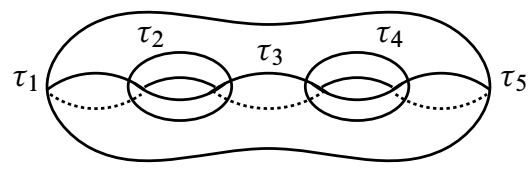

Figure 1: Standard generators of $\mathrm{Map}_{2}$

It is easy to see that the Lefschetz fibrations $\varphi_{1}$ and $\varphi_{2}$ are not isomorphic. For example, their monodromy groups are different: the monodromy of $\varphi_{1}$ surjects onto Map 2 , while that of $\varphi_{2}$ takes values in the subgroup of Map 2 generated by $\tau_{1}, \tau_{2}, \tau_{3}$ and $\tau_{4}$ (this is a proper subgroup since its image under the natural surjective homomorphism $\operatorname{Map}_{2} \rightarrow \mathfrak{S}_{6}$ mapping $\tau_{i}$ to $(i, i+1)$ is $\left.\mathfrak{S}_{5} \subsetneq \mathfrak{S}_{6}\right)$. However, this difference sheds very little light on the structure of $X_{1}$ and $X_{2}$ as symplectic 4-manifolds; in fact, it can be understood in terms of elementary topological considerations. We start with a remark.

Remark 2.10 As a smooth 4-manifold, $X_{1}$ can also be constructed as follows: in $\mathbb{C P}^{1} \times \mathbb{C P}^{1}$, consider a configuration of 6 "horizontal" lines $H_{i}=\mathbb{C P}^{1} \times\left\{b_{i}\right\}$ and 12 "vertical" lines $F_{j}=\left\{a_{j}\right\} \times \mathbb{C P}^{1}$; blow up $\mathbb{C P}^{1} \times \mathbb{C P}^{1}$ at the 72 intersection points $\left(a_{j}, b_{i}\right)$, and denote by $\widehat{H}_{i}$ and $\widehat{F}_{j}$ the proper transforms of the lines $H_{i}$ and $F_{j}$. Then $X_{1}$ is diffeomorphic to the double cover of the blowup of $\mathbb{C P}^{1} \times \mathbb{C P}^{1}$ branched along $\bigcup \hat{H}_{i} \cup \bigcup \widehat{F}_{j}$.

Indeed, this follows from simultaneous resolution of singularities: the double cover of $\mathbb{C P}^{1} \times \mathbb{C P}^{1}$ branched along the nodal configuration $\bigcup H_{i} \cup \bigcup F_{j}$ is a singular surface with 72 ordinary double points. The double points can be either smoothed, which amounts to smoothing of the branch curve in $\mathbb{C P}^{1} \times \mathbb{C P}^{1}$, or blown up, which amounts to blowing up $\mathbb{C P} P^{1} \times \mathbb{C P}^{1}$ and taking the proper transform of the branch curve. Even though these two constructions differ from a symplectic point of view (blowing up creates symplectic -2 -spheres, while smoothing creates Lagrangian $-2-$ spheres), the resulting 4-manifolds are diffeomorphic.

The same argument yields an alternative construction of $X_{2}$ as a double cover of a blow-up of $\mathbb{F}_{6}$.

The cohomology groups $H^{2}\left(X_{1}, \mathbb{Z}\right)$ and $H^{2}\left(X_{2}, \mathbb{Z}\right)$ contain rank 2 sublattices $\Lambda_{1}=$ $\pi_{1}^{*} H^{2}\left(\mathbb{C P} \mathbb{P}^{1} \times \mathbb{C P}^{1}, \mathbb{Z}\right)$ and $\Lambda_{2}=\pi_{2}^{*} H^{2}\left(\mathbb{F}_{6}, \mathbb{Z}\right)$. Even though the lattices $H^{2}\left(X_{1}, \mathbb{Z}\right)$ and $H^{2}\left(X_{2}, \mathbb{Z}\right)$ (equipped with the intersection pairings) are isomorphic, and the sublattices $\Lambda_{1}$ and $\Lambda_{2}$ (equipped with the restrictions of the intersection pairings) are also isomorphic, we claim that the pairs $\left(H^{2}\left(X_{i}, \mathbb{Z}\right), \Lambda_{i}\right)$ are not isomorphic. This implies: 
Proposition 2.11 There is no homeomorphism $h: X_{1} \rightarrow X_{2}$ such that $h^{*}\left(\Lambda_{2}\right)=\Lambda_{1}$.

Proof Take an element of $\Lambda_{1}$ of the form $\pi_{1}^{*}(p, q)$, where we implicitly identify $H^{2}\left(\mathbb{C P} \mathbb{P}^{1} \times \mathbb{C P} \mathbb{P}^{1}, \mathbb{Z}\right)$ with $\mathbb{Z}^{2}$. Recall the description of $X_{1}$ given in Remark 2.10, and consider the homology classes $A, B \in H_{2}\left(X_{1}, \mathbb{Z}\right)$ represented by the preimages of $\hat{H}_{1}$ and $\widehat{F}_{1}$. By construction, $\left\langle\pi_{1}^{*}(p, q), A\right\rangle=p$ and $\left\langle\pi_{1}^{*}(p, q), B\right\rangle=q$. This implies that, if $\pi_{1}^{*}(p, q)$ is divisible by 2 in $H^{2}\left(X_{1}, \mathbb{Z}\right)$, then $p$ and $q$ are both even, and hence $\pi_{1}^{*}(p, q)$ is also divisible by 2 in $\Lambda_{1}$.

On the other hand, let $\alpha \in H^{2}\left(\mathbb{F}_{6}, \mathbb{Z}\right)$ be the class Poincaré dual to the exceptional section $\Delta_{\infty}$, and consider $\pi_{2}^{*} \alpha \in \Lambda_{2}$ : for every class $[C] \in H_{2}\left(X_{2}, \mathbb{Z}\right)$, we have

$$
\left\langle\pi_{2}^{*} \alpha,[C]\right\rangle=\left\langle\alpha,\left(\pi_{2}\right)_{*}[C]\right\rangle=\left[\Delta_{\infty}\right] \cdot\left(\pi_{2}\right)_{*}[C]=2\left[\pi_{2}^{-1}\left(\Delta_{\infty}\right)\right] \cdot[C]
$$

(in the last equality we have used the fact that $\Delta_{\infty}$ is a component of the branch curve of $\left.\pi_{2}\right)$. This implies that $\pi_{2}^{*} \alpha$ is divisible by 2 in $H^{2}\left(X_{2}, \mathbb{Z}\right)$; however, $\alpha$ is primitive in $H^{2}\left(\mathbb{F}_{6}, \mathbb{Z}\right)$, so $\pi_{2}^{*} \alpha$ is not divisible by 2 in $\Lambda_{2}$. This completes the proof.

The fibers of $\varphi_{1}$ represent the class $\pi_{1}^{*}(0,1)$, while the fibers of $\varphi_{2}$ represent the class $\pi_{2}^{*}[F]$ where $[F]$ is the class of the fiber of $\mathbb{F}_{6}$. Moreover, the canonical classes of $X_{1}$ and $X_{2}$ are $c_{1}\left(K_{X_{1}}\right)=\pi_{1}^{*}(1,4)$ and $c_{1}\left(K_{X_{2}}\right)=\pi_{2}^{*}\left(\left[\Delta_{0}\right]+[F]\right)$. (Here we are implicitly using the isomorphism between homology and cohomology given by Poincaré duality).

If the two Lefschetz fibrations $\varphi_{1}$ and $\varphi_{2}$ were isomorphic then we would have a diffeomorphism $h: X_{1} \rightarrow X_{2}$ taking the fiber class to the fiber class. Moreover, $h$ would also map the canonical class to the canonical class (this follows eg from SeibergWitten theory, since $\pm K_{X_{i}}$ are the only basic classes, and evaluation on the fiber classes shows that the signs are preserved). Since the fiber classes and canonical classes generate $\Lambda_{1}$ and $\Lambda_{2}$, this contradicts Proposition 2.11.

Remark 2.12 These simple topological considerations are at the heart of the problem. Indeed, it is easy to distinguish $X_{1}$ and $X_{2}$ as complex surfaces because the projections $\pi_{1}, \pi_{2}$ and the lattices $\Lambda_{1}, \Lambda_{2}$ are naturally determined by the complex geometry of the Horikawa surfaces: they can, for example, be interpreted in terms of the canonical linear systems, or in terms of algebraic vanishing cycles for nodal degenerations. If there were a purely symplectic construction allowing us to characterize the lattices $\Lambda_{1}$ and $\Lambda_{2}$ in terms of the symplectic topology of $X_{1}$ and $X_{2}$ (without using the extra data provided by the coverings $\pi_{i}$ ), then it would follow that $X_{1}$ and $X_{2}$ are different symplectic 4-manifolds. Donaldson has suggested that one should compare the sets of homology classes realized by embedded Lagrangian spheres in $X_{1}$ and $X_{2}$; 
it is conjectured that these coincide with algebraic vanishing cycles, and hence span precisely the orthogonal complements to $\Lambda_{1}$ and $\Lambda_{2}$ (see Section 8 for more on this topic). However, to this date little progress has been made in this direction.

\subsection{Luttinger surgery and partial twistings}

We now discuss some properties of braiding constructions and Luttinger surgery in the context of Lefschetz pencils and double covers. The reader is referred to [2] for more background on these topics (see also [9]).

Consider a 2:1 covering $\pi: X \rightarrow Y$ of symplectic 4-manifolds, branched along a smooth symplectic curve $C \subset Y$. Assume that we are given a Lagrangian annulus $A$ with interior in $Y \backslash C$ and boundary contained in $C$. Then we can obtain a new symplectic curve $C^{\prime} \subset Y$ by braiding the curve $C$ along the annulus $A$, in the manner depicted on Figure 2. Namely, we cut out a neighborhood $U$ of $A$, and glue it back via a non-trivial diffeomorphism which interchanges two of the connected components of $C \cap \partial U$, in such a way that the product of $S^{1}$ with the trivial two-strand braid is replaced by the product of $S^{1}$ with a half-twist (see [2] for details).

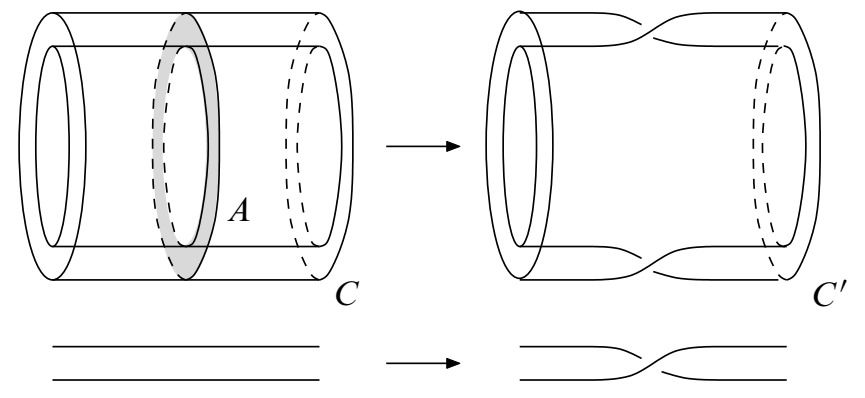

Figure 2: Braiding a branch curve

Braiding the curve $C$ along the Lagrangian annulus $A$ affects the branched cover $X$ by a Luttinger surgery along the smooth embedded Lagrangian torus $T=\pi^{-1}(A)$ [2]. This operation consists of cutting out from $X$ a tubular neighborhood of $T$, foliated by parallel Lagrangian tori, and gluing it back via a symplectomorphism wrapping the meridian around the torus (in the direction of the preimage of an arc joining the two boundaries of $A$ ), while the longitudes are not affected.

Here we are specifically interested in the situation already considered in Section 2.2 , namely when we are given a Lefschetz pencil (or fibration) $f: Y \backslash B \rightarrow \mathbb{C P}^{1}$ with respect to which $C$ lies in a generic position. Assume that we have a loop $\gamma \in \pi_{1}\left(\mathbb{C} \mathbb{P}^{1} \backslash \operatorname{crit}(f)\right)$ along which the monodromy of $f$ is trivial, and that $C$ is 
transverse to the fibers of $f$ in a neighborhood of $f^{-1}(\gamma)$. Also assume for now that the braid monodromy of $C$ along $\gamma$ is trivial.

Consider an arc $\eta$ in the fiber $\Sigma$ of $f$ above a point of $\gamma$, with end points $x^{\prime}, x^{\prime \prime} \in \Sigma \cap C$. We can locally identify $Y$ with a product $V_{1} \times V_{2}$, where $V_{1}$ is a neighborhood of $\gamma$ in $\mathbb{C P}^{1} \backslash \operatorname{crit}(f)$ (such that $C$ is transverse to the fibers of $f$ over $V_{1}$ ), and $V_{2}$ is a neighborhood of $\eta$ in $\Sigma$. In this local model, we can assume that $f$ is the projection to the first factor $V_{1}$, and that $C$ is the subset $V_{1} \times\left\{x^{\prime}, x^{\prime \prime}\right\} \subset V_{1} \times V_{2}$. Consider the annulus $A=\gamma \times \eta$ with boundary in $C$. Standard results about symplectic structures on Lefschetz fibrations (see eg $[12 ; 13]$ ) imply that, up to a small perturbation of the symplectic form, we can assume the annulus $A$ to be Lagrangian. We can then braid $C$ along $A$ as described above, to obtain a new symplectic curve $C^{\prime} \subset Y$ which coincides with $C$ outside of $V_{1} \times V_{2}$ and is transverse to the fibers of $f$ inside $V_{1} \times V_{2}$. (In fact, the construction can often be carried out without perturbing the symplectic form, provided the annulus $A$ is "thin" enough and the neighborhood $V_{1}$ can be chosen large enough to allow $C$ to be distorted in the direction of $\eta$ while remaining symplectic).

The braid monodromy of $C^{\prime}$ differs from that of $C$ by a partial conjugation operation. Namely, let $\mathrm{D}_{ \pm}$be the two components of $\mathbb{C P}^{1} \backslash V_{1}$, and choose the base point in $\mathbb{C P}^{1} \backslash \operatorname{crit}(f)$ to lie on the boundary of $\mathrm{D}_{+}$. Then $C$ and $C^{\prime}$ have the same braid monodromy along any loop in $\mathrm{D}_{+}$, but their braid monodromies around points of $\mathrm{D}_{-}$ differ by conjugation by the half-twist $\sigma_{\eta}$ along $\eta$. In other terms, the vanishing arcs corresponding to the vertical tangencies of $C$ inside $f^{-1}$ (D_) are replaced by their images under $\sigma_{\eta}$.

Since we have only used the local structure near the annulus $A$, we can in fact relax our assumption concerning the braid monodromy of $C$ along $\gamma$ : it is sufficient to assume that this braid monodromy fixes the arc $\eta$ (ie, that it can be realized by an isotopy of $\Sigma$ supported in the complement of $V_{2}$. Similarly, we do not have to assume that the monodromy of $f$ along $\gamma$ is trivial, we only need to assume triviality in the considered portion of the fiber (however, we will only consider situations in which the monodromy of $f$ along $\gamma$ is trivial away from the boundary of $\Sigma$ ).

Now consider the double covers $\pi: X \rightarrow Y$ and $\pi^{\prime}: X^{\prime} \rightarrow Y$ branched along $C$ and $C^{\prime}$, and the Lefschetz pencils $\tilde{f}=f \circ \pi$ and $\tilde{f}^{\prime}=f \circ \pi^{\prime}$. By the result of [2], these differ by a Luttinger surgery along the Lagrangian torus $T=\pi^{-1}(A)$, performed in the direction of the loop $\delta=\pi^{-1}(\eta) \subset \widetilde{\Sigma}$.

The monodromies of $\tilde{f}$ and $\tilde{f}^{\prime}$ differ by a partial conjugation:

Proposition 2.13 The Lefschetz pencils $\tilde{f}$ and $\tilde{f}^{\prime}$ have the same monodromy along any loop in $\mathrm{D}_{+}$, but their monodromies around critical values in $\mathrm{D}_{-}$differ by conjugation by the Dehn twist along $\delta$. 
Proof The result follows directly from Proposition 2.8. Indeed, it is clear that the monodromies over $\mathrm{D}_{+}$coincide. If we consider a vertical tangency of $C$ in $f^{-1}$ (D_), the braiding operation replaces the vanishing arc by its image under $\sigma_{\eta}$; since the lifting homomorphism maps $\sigma_{\eta}$ to the Dehn twist $t_{\delta}$, the corresponding vanishing cycles of $\tilde{f}$ and $\tilde{f}^{\prime}$ differ precisely by $t_{\delta}$. Next, consider a critical point of $f$ in $f^{-1}\left(D_{-}\right)$. Braiding is only a modification of the curve $C$, so the vanishing cycles of $f$ are not affected, and neither are the corresponding vanishing cycles of $\widetilde{f}$. However, viewing the half-twist $\sigma_{\eta}$ as an isotopy of $\Sigma$ supported in $V_{2}$, and observing that $\left[\sigma_{\eta}\right]$ is the identity element in the mapping class group of $\Sigma$, we can also replace each vanishing cycle of $f$ by its image under $\sigma_{\eta}$; after lifting, the corresponding vanishing cycles of $\widetilde{f}^{\prime}$ become the images of those of $\tilde{f}$ under $t_{\delta}$ (which, in fact, acts trivially).

Another way to interpret these constructions is in terms of twisted fiber sums. Namely, $Y$ can be obtained by gluing $Y_{+}=f^{-1}\left(\mathrm{D}_{+}\right)$and $Y_{-}=f^{-1}\left(\mathrm{D}_{-}\right)$along their boundary via a diffeomorphism $\psi: \partial Y_{+} \rightarrow \partial Y_{-}$compatible with the Lefschetz fibrations $f_{ \pm}=f_{\mid Y_{ \pm}}$; the branch curve $C$ is also obtained by gluing $C_{ \pm}=C \cap f^{-1}\left(\mathrm{D}_{ \pm}\right)$along their boundaries via the diffeomorphism $\psi$. This realizes $(Y, C)$ as the pairwise fiber sum of $\left(Y_{+}, C_{+}\right)$and $\left(Y_{-}, C_{-}\right)$. If we instead glue $\left(Y_{+}, C_{+}\right)$to $\left(Y_{-}, C_{-}\right)$by the diffeomorphism obtained by composing $\psi$ with the half-twist $\sigma_{\eta}$ inside each fiber of $f$ above the boundary (abusing notation we denote this diffeomorphism by $\sigma_{\eta} \circ \psi$ ), we obtain the pair $\left(Y, C^{\prime}\right)$, realized as the twisted fiber sum of $\left(Y_{+}, C_{+}\right)$and $\left(Y_{-}, C_{-}\right)$.

Passing to the double covers, we can view $X$ as a fiber sum $X_{+} \cup \tilde{\psi} X_{-}$, where $X_{ \pm}=\pi^{-1}\left(Y_{ \pm}\right)$and $\tilde{\psi}$ is a fiber-preserving diffeomorphism which lifts $\psi$. In this language, the Lefschetz pencil $\tilde{f}^{\prime}$ on $X^{\prime}$ is the twisted fiber sum $X_{+} \cup \tilde{\psi}^{\prime} X_{-}$of $\widetilde{f}_{+}=\widetilde{f}_{\mid X_{+}}$and $\widetilde{f}_{-}=\widetilde{f}_{\mid X_{-}}$, where $\widetilde{\psi}^{\prime}=t_{\delta} \circ \tilde{\psi}$ is obtained by composing $\widetilde{\psi}$ with the Dehn twist $t_{\delta}$ inside each fiber of $\tilde{f}$. We also say that $\tilde{f}^{\prime}$ is a "partial twisting" of $\tilde{f}$.

\section{The monodromy of the canonical pencil on $X_{2}$}

The goal of this section is to compute the monodromy of a canonical pencil of curves on the Horikawa surface $X_{2}$ (expressed as a collection of 196 Dehn twists in Map 17,16 ). The reader who does not care about details of the setup and calculations may skip directly ahead to Section 3.4, where the final formula is given along with the necessary notations.

\subsection{A special configuration}

Recall that $X_{2}$ is the double cover of $\mathbb{F}_{6}$ branched along $\Delta_{\infty} \cup C_{2}$, where $\Delta_{\infty}$ is the exceptional section and $C_{2}$ is a smooth curve in the linear system $\left|5 \Delta_{0}\right|$. Since 
$K_{X_{2}}=\pi_{2}^{*}\left(\left[\Delta_{0}\right]+[F]\right)$, we can obtain a pencil of curves in the linear system $\left|K_{X_{2}}\right|$ on $X_{2}$ by taking the preimages of a pencil of curves in the linear system $\left|\Delta_{0}+F\right|$ on $\mathbb{F}_{6}$.

The connectedness of the space of generic configurations (which is the complement of a divisor in some projective variety) implies that the topology of the resulting pencil of curves on $X_{2}$ does not depend on the choices made, as long as the curve $C_{2}$ and the chosen pencil on $\mathbb{F}_{6}$ are in general position with respect to each other. Accordingly, we will choose a particular configuration for which the monodromy calculations are manageable.

The Hirzebruch surface $\mathbb{F}_{6}=\mathbb{P}(\mathcal{O} \oplus \mathcal{O}(6))$ can be thought of as a fiberwise compactification of the line bundle $\mathcal{O}(6)$ over $\mathbb{C P}^{1}$; in this sense, $\Delta_{0}$ is the zero section, and $\Delta_{\infty}$ is the section at infinity. Moreover, we think of $\mathbb{C P}^{1}$ as $\mathbb{C} \cup\{\infty\}$, and trivialize $\mathcal{O}(6)$ over $\mathbb{C}$ by means of a holomorphic section vanishing with order 6 at infinity. In this trivialization, sections of $\mathcal{O}(6)$ are represented by polynomials of degree at most 6 in one complex variable.

We will be considering a pencil of curves in the linear system $\left|\Delta_{0}+F\right|$, with base points $p_{1}, \ldots, p_{8} \in \mathbb{F}_{6}$; this pencil can be viewed equivalently as a family of curves $\Sigma_{\alpha} \subset \mathbb{F}_{6}, \alpha \in \mathbb{C P}^{1}$, or as a map $f: \mathbb{F}_{6} \backslash\left\{p_{1}, \ldots, p_{8}\right\} \rightarrow \mathbb{C P}^{1}$. We choose the base points $p_{1}=\left(z_{1}, 0\right), \ldots, p_{7}=\left(z_{7}, 0\right)$ on the zero section, and $p_{8}=\left(z_{8}, \epsilon\right)$ close to the zero section ( $\epsilon$ is a small nonzero constant). We will set things up in such a way that all the interesting phenomena happen in the real part of $\mathbb{F}_{6}$ (thus making it easier to visualize the monodromy). Accordingly, we choose the constants $z_{1}, \ldots, z_{8}, \epsilon$ to be real numbers, with $z_{1}<\cdots<z_{8}$ and $\epsilon<0$.

Each curve of the pencil through $p_{1}, \ldots, p_{8}$ intersects $\Delta_{\infty}$ in exactly one point, and so we can parameterize the pencil by its restriction to $\Delta_{\infty}$ : namely, for each $\alpha \in \mathbb{C P}^{1}$, we call $\Sigma_{\alpha}$ the curve in the pencil which passes through the point $(\alpha, \infty) \in \Delta_{\infty}$. If $\Sigma_{\alpha}$ is smooth, then it can be viewed as the graph of a meromorphic section $s_{\alpha}$ of $\mathcal{O}(6)$ with a simple pole at $\alpha$ and zeroes at $z_{1}, \ldots, z_{7}$; in the given trivialization of $\mathcal{O}(6)$, this section is given by the formula

$$
s_{\alpha}(z)=\epsilon^{\prime} \frac{z_{8}-\alpha}{z-\alpha} \prod_{i=1}^{7}\left(z-z_{i}\right),
$$

where $\epsilon^{\prime}=\epsilon / \prod\left(z_{8}-z_{i}\right)$. By projecting to $\mathbb{C P}^{1}$, we can identify each smooth fiber of $f$ (ie, $\Sigma_{\alpha}$ with the base points removed) with $\mathbb{C P}^{1} \backslash\left\{z_{1}, \ldots, z_{8}\right\}$. Accordingly, the monodromy of $f$ takes values in the mapping class group Map, $\mathrm{p}_{0,8}$ of $\mathbb{C P}^{1}$ with small discs around each $z_{i}$ removed.

It is easy to check that $f$ has 8 singular fibers, corresponding to the values $\alpha=$ $z_{1}, \ldots, z_{8}$; for $\alpha=z_{i}$, the curve $\Sigma_{\alpha}$ consists of two components: the fiber of $\mathbb{F}_{6}$ over 
$z_{i}$, and the unique curve in $\left|\Delta_{0}\right|$ passing through all $p_{j}, j \neq i$ (for $i=8$ this is the zero section). The base point $p_{i}$ lies in the fiber component, and the seven other base points lie in the section component; hence, the vanishing cycle of $f$ at $\alpha=z_{i}$ is a boundary curve (a circle separating $z_{i}$ from the other punctures).

Choosing $\epsilon$ small enough ensures that, outside of a fixed neighborhood of $\Delta_{0}$, the pencil of curves $\left(\Sigma_{\alpha}\right)_{\alpha \in \mathbb{C P}^{1}}$ is arbitrarily close to the standard fibration $\mathbb{F}_{6} \rightarrow \mathbb{C P}^{1}$. In fact, each $\Sigma_{\alpha}$ lies in a small neighborhood of $\Delta_{0} \cup\left(\{\alpha\} \times \mathbb{C P}^{1}\right)$; therefore, by choosing the curve $C_{2}$ (the "main" component of the branch curve of $\pi_{2}$ ) transverse to the zero section, we can obtain an explicit description of its behavior with respect to the pencil $f$.

Choose real numbers $q_{1}, \ldots, q_{6}$ and $r_{1}, \ldots, r_{6}$ such that

$$
q_{1}<z_{1}<r_{1}<q_{2}<z_{2}<r_{2}<\cdots<q_{6}<z_{6}<r_{6}<z_{7}<z_{8},
$$

and consider the graph of the holomorphic section $u(z)=\prod_{i=1}^{6}\left(z-q_{i}\right)$ of $\mathcal{O}(6)$. We can construct nearby curves in the real part of the pencil of curves in $\left|\Delta_{0}\right|$, passing through the six points $\left(r_{i}, u\left(r_{i}\right)\right)$, by considering the graphs of holomorphic sections of $\mathcal{O}(6)$ of the form

$$
u(z)+\lambda \prod_{i=1}^{6}\left(z-r_{i}\right),
$$

with $\lambda \in \mathbb{R}$ small. Consider five such holomorphic sections $u_{1}, \ldots, u_{5}$ of $\mathcal{O}(6)$ : their graphs $\Gamma_{1}, \ldots, \Gamma_{5}$ intersect each other transversely at $\left(r_{i}, u\left(r_{i}\right)\right)(1 \leq i \leq 6)$, and intersect the zero section transversely at real points near $q_{1}, \ldots, q_{6}$. We define $\Gamma_{j} \cap \Delta_{0}=\left\{q_{1, j}, \ldots, q_{6, j}\right\}$, with $q_{i, j} \approx q_{i}$. It is easy to check that the various points $q_{i, j}$ are in the same order near each $q_{i}$, so we can assume that $q_{i, 1}<\cdots<q_{i, 5}$ for all $i$. Choosing the perturbations small enough, we can also assume that $q_{i, 1}>r_{i-1}$ and $q_{i, 5}<z_{i}$.

The configuration $\Gamma_{1} \cup \cdots \cup \Gamma_{5}$ can be smoothed to a nearby curve in the linear system $\left|5 \Delta_{0}\right|$, which we take as our choice for $C_{2}$. Since this smoothing can be realized by an arbitrarily small perturbation, we can ensure that $C_{2}$ is contained in an arbitrarily small neighborhood of $\bigcup \Gamma_{i}$, and arbitrarily $C^{1}$-close to $\bigcup \Gamma_{i}$ outside of an arbitrarily small neighborhood of the points $\left(r_{i}, u\left(r_{i}\right)\right)$. Moreover, we choose the smoothing perturbation to be real and generic, so that $C_{2}$ is defined by an equation with real coefficients, and its tangencies with the fibers of $\mathbb{F}_{6}$ are nondegenerate, real, and lie in distinct fibers near $r_{i}$. Finally, the points of $C_{2} \cap \Delta_{0}$ lie arbitrarily close to those of $\Gamma_{j} \cap \Delta_{0}$; changing our notation, we will again call them $q_{i, j}, 1 \leq i \leq 6,1 \leq j \leq 5$, and observe that we still have $r_{i-1}<q_{i, 1}<\cdots<q_{i, 5}<z_{i}$. Our choices for $C_{2}$ and $\Sigma_{\alpha}$ are summarized on Figure 3 . 


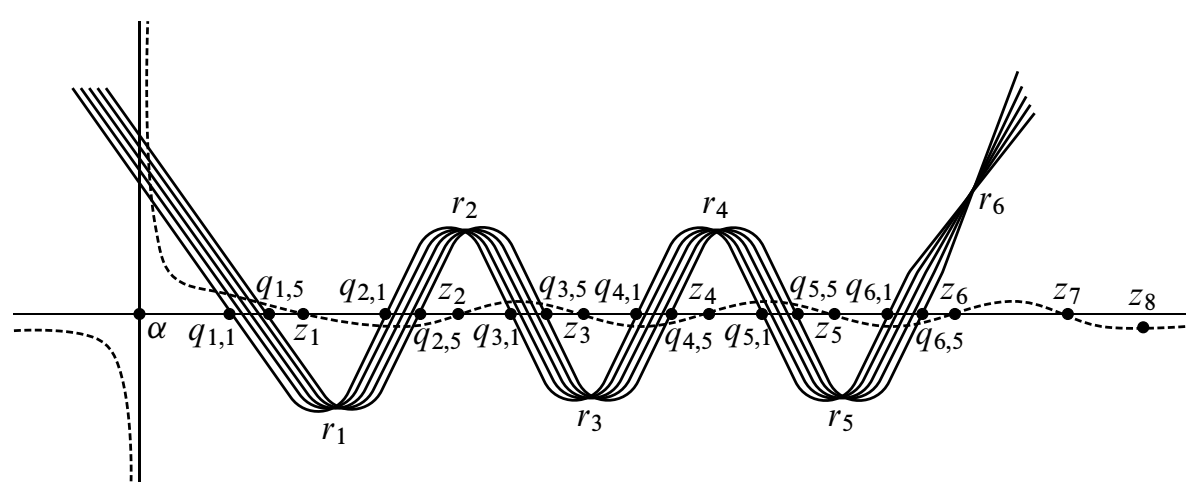

Figure 3: The curves $C_{2}$ (solid) and $\Sigma_{\alpha}$ (dashed) in $\mathbb{F}_{6}$

\subsection{More notations and conventions}

We need to study the braid monodromy of the branch curve $\Delta_{\infty} \cup C_{2}$ with respect to the pencil $f$, ie, the manner in which the intersection points of $\Delta_{\infty} \cup C_{2}$ with $\Sigma_{\alpha}$ depend on the choice of $\alpha$. Recall that we can trivialize the pencil $f$ (except at its singular fibers) by using the standard projection $p r: \mathbb{F}_{6} \rightarrow \mathbb{C P}^{1}$, which allows us to identify the complement of the base points in $\Sigma_{\alpha}$ with $\mathbb{C P}^{1} \backslash\left\{z_{1}, \ldots, z_{8}\right\}$.

Assume that $\alpha$ is not too close to any of the special values $z_{i}, q_{i}, r_{i}$. Then the projections to $\mathbb{C P}^{1} \backslash\left\{z_{1}, \ldots, z_{8}\right\}$ of the 36 points where $\Sigma_{\alpha}$ intersects $\Delta_{\infty} \cup C_{2}$ all lie in a small neighborhood of $\left\{\alpha, q_{1}, \ldots, q_{6}\right\}$, and can be labelled in a simple manner according to their respective positions:

- the intersection between $\Sigma_{\alpha}$ and $\Delta_{\infty}$ takes place at $\alpha$.

- the 5 intersections between $C_{2}$ and the "vertical" part of $\Sigma_{\alpha}$ take place at $\widetilde{\alpha}_{1}, \ldots, \widetilde{\alpha}_{5}$ in a neighborhood of $\alpha$. We label them in such a way that, upon deforming $C_{2}$ back to the nearby singular configuration $\Gamma_{1} \cup \cdots \cup \Gamma_{5}, \tilde{\alpha}_{i}$ corresponds to an intersection of $\Sigma_{\alpha}$ with $\Gamma_{i}$.

- the 30 intersections between $C_{2}$ and the "horizontal" part of $\Sigma_{\alpha}$ take place at $\widetilde{q}_{1,1}, \ldots, \widetilde{q}_{6,5}$, where each $\widetilde{q}_{i, j}$ is close to $q_{i, j}$.

In order to define braid monodromy and vanishing arcs, we need to fix a reference fiber of the pencil $f$, ie, some reference value $\alpha_{0}$, and arcs from this reference value to the various values of $\alpha$ for which $\Delta_{\infty} \cup C_{2}$ is tangent to $\Sigma_{\alpha}$. We choose the reference value $\alpha_{0}$ to be a sufficiently negative real number, so that $\alpha_{0} \ll q_{1,1}$. The respective positions of $\Sigma_{\alpha_{0}}$ and $\Delta_{\infty} \cup C_{2}$ are then as pictured on Figure 3. In particular, the 
images under the projection from $\Sigma_{\alpha_{0}}$ to $\mathbb{C P}^{1}$ of the 36 intersection points and the 8 base points are all real, and in the order

$$
\begin{array}{r}
\alpha<\tilde{\alpha}_{5}<\cdots<\tilde{\alpha}_{1}<\tilde{q}_{1,1}<\cdots<\widetilde{q}_{1,5}<z_{1}<\tilde{q}_{2,1}<\cdots<\tilde{q}_{2,5}<z_{2}<\ldots \\
\cdots<z_{5}<\widetilde{q}_{6,1}<\cdots<\widetilde{q}_{6,5}<z_{6}<z_{7}<z_{8} . \\
\end{array}
$$

To determine the braid monodromy of $\Delta_{\infty} \cup C_{2}$, we consider what happens to these various intersections as the value of $\alpha$ increases along the real axis from $\alpha_{0}$ to a large positive value. As we will see below, there are in total 180 values of $\alpha$ for which the curve $\Sigma_{\alpha}$ is tangent to $C_{2}$, in addition to the 8 values of $\alpha$ for which $\Sigma_{\alpha}$ is nodal. Our convention will be that we determine the monodromy around each critical value $\alpha_{c r, i}$ by considering a loop in $\pi_{1}\left(\mathbb{C} \backslash\left\{\alpha_{c r, j}\right\}, \alpha_{0}\right)$ constructed as follows: choose a point $\alpha_{i}^{\prime}$ on the real axis just to the left of the critical value $\alpha_{c r, i}$, and an arc $\eta_{i}$ joining $\alpha_{0}$ to $\alpha_{i}^{\prime}$ inside the upper half-plane (ie, passing above all the critical values between $\alpha_{0}$ and $\alpha_{c r, i}$ ); then we consider the loop $\gamma_{i}$ obtained by composing the arc $\eta_{i}$ from $\alpha_{0}$ to $\alpha_{i}^{\prime}$, a small circle around $\alpha_{c r, i}$ (counterclockwise), and the arc $\eta_{i}^{-1}$ back to $\alpha_{0}$. This choice ensures in particular that, if we order the critical values $\alpha_{c r, i}$ in increasing order along the real axis, the loops $\gamma_{i}$ form an ordered collection of generators for $\pi_{1}\left(\mathbb{C} \backslash\left\{\alpha_{c r, j}\right\}, \alpha_{0}\right)$.

Our calculation of braid monodromy relies on the following ingredients:

(1) The configuration of intersection points for $\alpha=\alpha_{i}^{\prime}$ determines readily the vanishing arc at $\alpha_{c r, i}$ : namely, in the nearby fiber $\Sigma_{\alpha_{i}^{\prime}}$ the vanishing arc is simply a straight line segment joining the two intersection points which approach each other as $\alpha \rightarrow \alpha_{c r, i}$.

(2) The vanishing arc in the reference fiber $\Sigma_{\alpha_{0}}$ is obtained from the local configuration in $\Sigma_{\alpha_{i}^{\prime}}$ by transporting along the arc $\eta_{i}^{-1}$, or equivalently, along a succession of counterclockwise half-circles around all the critical values $\alpha_{c r, j}<\alpha_{c r, i}$. (As a general principle, the main feature is that the intersection points labelled $\alpha, \widetilde{\alpha}_{5}, \ldots, \widetilde{\alpha}_{1}$ are moved counterclockwise back to the leftmost positions, since these intersection points stay close to $\alpha$ while the others remain close to $\left.q_{1}, \ldots, q_{5}\right)$.

(3) The configuration of intersection points for a value of $\alpha$ on the real axis just to the right of $\alpha_{c r, i}$ can be deduced from the configuration at $\alpha=\alpha_{i}^{\prime}$ by applying the "square root" of the monodromy clockwise around $\alpha_{c r, i}$, namely a clockwise 90 degree rotation of the two end points of the local vanishing arc. 


\subsection{The braid monodromy}

Upon increasing $\alpha$ along the real axis, the first critical values encountered lie near $q_{1}$. The braid monodromy near $q_{1}$ can be understood by following the approach outlined above. The real part of the local configuration looks as in Figure 4 (a translating hyperbola passing through five parallel lines); in particular, near $q_{1}$ there are 10 values $\alpha_{c r, 1}<\cdots<\alpha_{c r, 10}$ of $\alpha$ for which $\Sigma_{\alpha}$ is tangent to $C_{2}$.

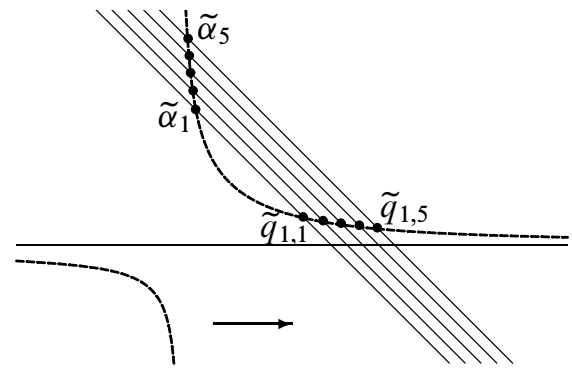

Figure 4: The configuration near $q_{1}$

The local configurations of the intersection points in the fibers immediately to the left of each critical value $\alpha_{c r, 1}, \ldots, \alpha_{c r, 10}$ are shown in Figure 5 (left), together with the corresponding vanishing arcs. Transporting these vanishing arcs back to the reference fiber $\Sigma_{\alpha_{0}}$ (by going counterclockwise around the previous critical values) yields the vanishing arcs represented in Figure 5 (right), which determine the braid monodromy of $\Delta_{\infty} \cup C_{2}$ near $q_{1}$.

The product of the 10 half-twists along these vanishing arcs is the braid which translates the disc D containing $\alpha, \widetilde{\alpha}_{5}, \ldots, \widetilde{\alpha}_{1}$ counterclockwise around the points $\widetilde{q}_{1,1}, \ldots, \widetilde{q}_{1,5}$ by 360 degrees, while simultaneously rotating the interior of $D$ clockwise by 360 degrees, and rotating the interior of the smaller disc $\mathrm{D}^{\prime}$ containing $\tilde{\alpha}_{5}, \ldots, \widetilde{\alpha}_{1}$ clockwise by another 360 degrees. This can be checked either by direct calculation, or more geometrically by analyzing the behavior of $\alpha, \widetilde{\alpha}_{5}, \ldots, \widetilde{\alpha}_{1}$ as $\alpha$ moves along the boundary of a larger circle enclosing $q_{1,1}, \ldots, q_{1,5}$ (it is clear that $\mathrm{D}$ is moved counterclockwise around $\widetilde{q}_{1,1}, \ldots, \widetilde{q}_{1,5}$; a careful analysis of the respective positions of the $\tilde{\alpha}_{i}$ relatively to $\alpha$ shows that the motion within $\mathrm{D}$ is as claimed).

The next contribution to monodromy occurs at $\alpha=z_{1}$; the curve $\Sigma_{\alpha}$ is then reducible, with one component (the fiber of $\mathbb{F}_{6}$ above $z_{1}$ ) containing the intersection points labelled $\alpha, \widetilde{\alpha}_{5}, \ldots, \widetilde{\alpha}_{1}$ and the base point $z_{1}$, while the other component contains all the other intersection points and base points. Hence, in a nearby smooth fiber the vanishing cycle is a simple closed curve around the points $\widetilde{\alpha}_{5}, \ldots, \widetilde{\alpha}_{1}, \alpha, z_{1}$ (which are 


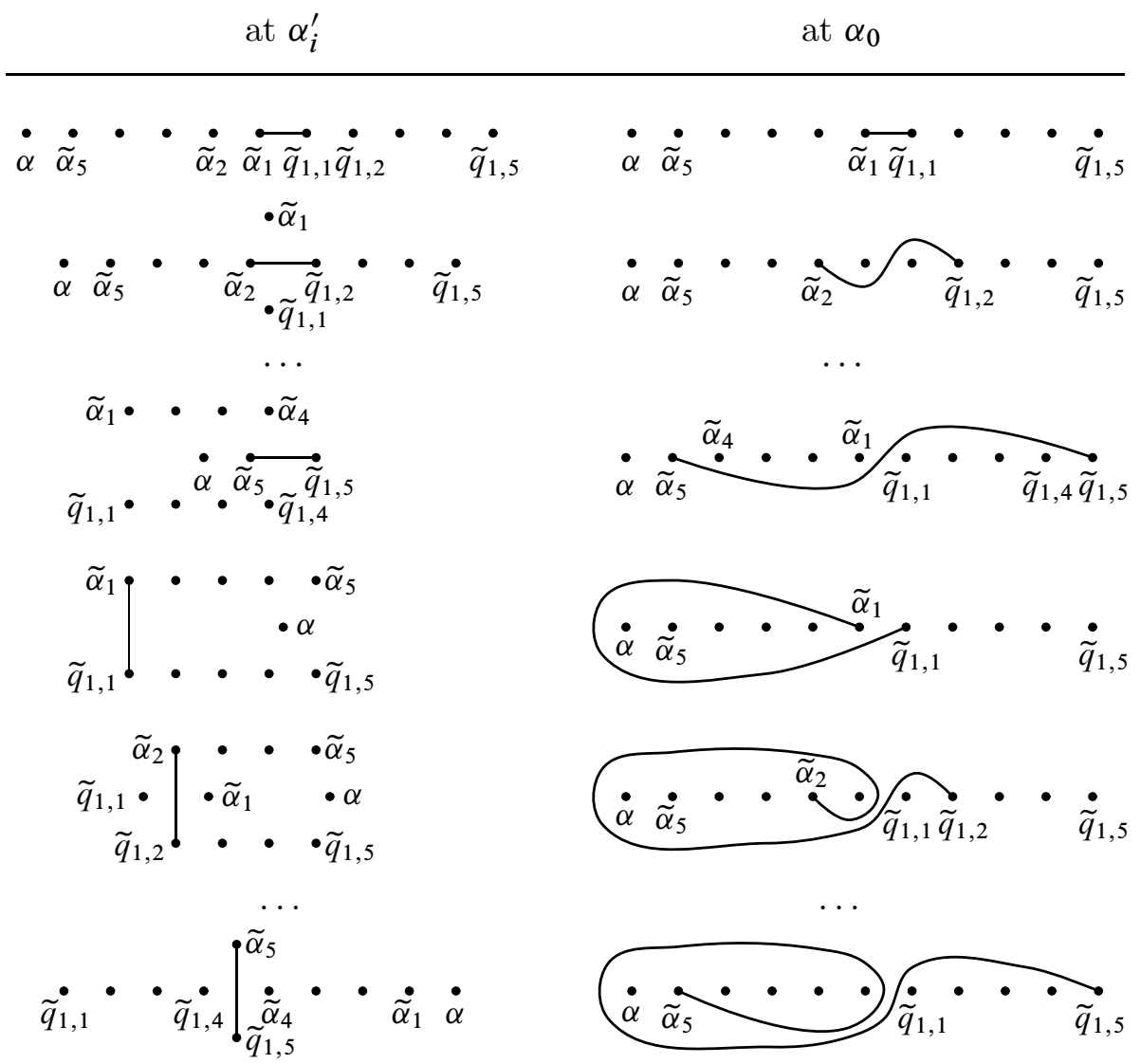

Figure 5: The vanishing arcs near $q_{1}$

adjacent in that order along the real axis). Transporting things back to the reference fiber $\Sigma_{\alpha_{0}}$ in the prescribed manner, using the square root of the monodromy around $q_{1}$ determined above, yields the vanishing cycle represented in Figure 6.

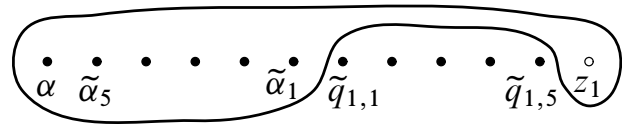

Figure 6: The vanishing cycle at $z_{1}$

Next, we consider the monodromy near $\alpha=r_{1}$. Because the point $\left(r_{1}, u\left(r_{1}\right)\right)$ lies away from the zero section, the tangencies between $C_{2}$ and $\Sigma_{\alpha}$ occur in the portion of $\Sigma_{\alpha}$ which lies close to the fiber $\{\alpha\} \times \mathbb{C P}^{1}$. In particular, the braid monodromy consists of half-twists supported in a small disc containing the points $\widetilde{\alpha}_{1}, \ldots, \widetilde{\alpha}_{5}$, and 
can be understood in terms of a local model in which a moving fiber intersects a degree 5 curve obtained by smoothing a configuration of five concurrent lines.
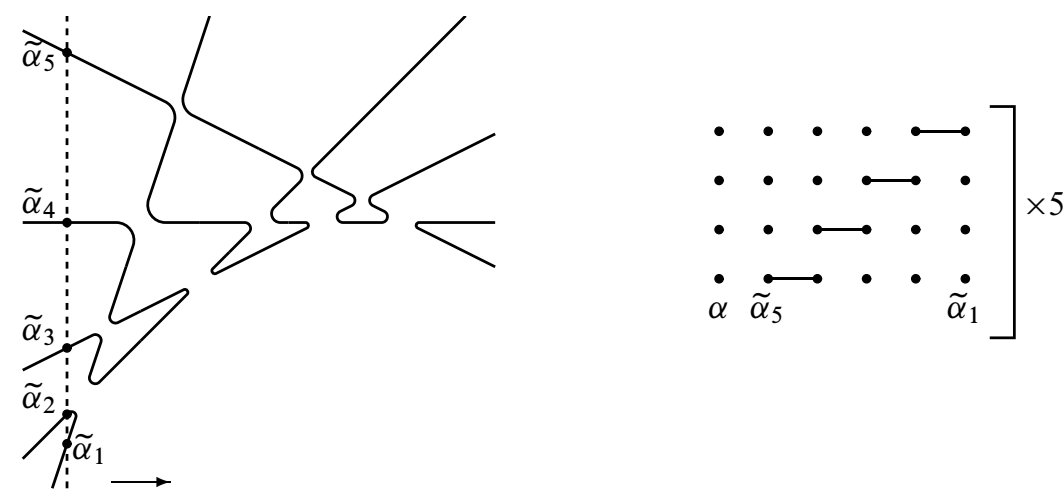

Figure 7: The monodromy near $r_{1}$

Hence, in a neighborhood of $r_{1}$, the braid monodromy of $\Delta_{\infty} \cup C_{2}$ with respect to the pencil $f$ coincides with the braid monodromy of a smooth algebraic plane curve of degree 5 with respect to a generic linear projection. The braid monodromy of smooth algebraic plane curves has been studied extensively, and there are various well-known formulas (Hurwitz equivalent to each other, of course); see eg [21]. For completeness, we outline one possible approach: first deform the curve so that it lies close to a configuration of five lines in general position, as in Figure 7. Consistently with the choice we have made so far, consider loops that reach each critical value $\alpha_{c r, i}$ via an arc in the upper half-plane. In that case, the braid monodromy can be read off from Figure 7 using the same method as previously; the braid monodromy factorization that arises in this way is a product of 20 half-twists,

$$
\prod_{i=1}^{4} \prod_{j=i+1}^{5}\left(\sigma_{i, j} \cdot \sigma_{i, j}\right),
$$

where $\sigma_{i j}$ is the half-twist along an arc that joins $\tilde{\alpha}_{i}$ to $\tilde{\alpha}_{j}$ passing below the real axis (see [21] for a careful derivation of this formula). However, it is well-known (see eg [21]) that this expression is Hurwitz equivalent to the simpler expression

$$
\left(\sigma_{1,2} \cdot \sigma_{2,3} \cdot \sigma_{3,4} \cdot \sigma_{4,5}\right)^{5}
$$

In other terms, if we change our choice of ordered collection of generators for $\pi_{1}(\mathbb{C} \backslash$ $\left.\left\{\alpha_{c r, j}\right\}, \alpha_{0}\right)$, we can assume that the 20 vanishing arcs near $r_{1}$ are as pictured in Figure 7 (right). 
The product of all the monodromies encountered so far (near $q_{1}, z_{1}$ and $r_{1}$ ) is simply the braid which moves the disc $\mathrm{D}$ containing $\alpha, \widetilde{\alpha}_{5}, \ldots, \widetilde{\alpha}_{1}$ by 360 degrees counterclockwise around the points $\widetilde{q}_{1,1}, \ldots, \widetilde{q}_{1,5}, z_{1}$. Moreover, for a real value of $\alpha$ such that $r_{1} \ll \alpha \ll q_{2}$, the intersections of $\Sigma_{\alpha}$ with $\Delta_{\infty} \cup C_{2}$ are all real, and in the order

$$
\begin{array}{r}
\tilde{q}_{1,1}<\cdots<\widetilde{q}_{1,5}<z_{1}<\alpha<\widetilde{\alpha}_{5}<\cdots<\widetilde{\alpha}_{1}<\widetilde{q}_{2,1}<\cdots<\widetilde{q}_{2,5}<z_{2}<\ldots \\
\cdots<z_{5}<\widetilde{q}_{6,1}<\cdots<\widetilde{q}_{6,5}<z_{6}<z_{7}<z_{8} .
\end{array}
$$

Hence, the local pictures near $q_{2}, z_{2}$ and $r_{2}$ are exactly the same as near $q_{1}, z_{1}$ and $r_{1}$ respectively (except that the configurations are flipped in the vertical direction, which does not change anything since we characterize intersection points in terms of their projections to the horizontal direction). When we transport the local monodromies back to the reference fiber $\Sigma_{\alpha_{0}}$ by an arc that goes counterclockwise around $q_{1}, z_{1}$ and $r_{1}$, the points $\alpha, \widetilde{\alpha}_{5}, \ldots, \widetilde{\alpha}_{1}$ are moved back to the left of $\widetilde{q}_{1,1}, \ldots, \widetilde{q}_{1,5}, z_{1}$ by a 180 degree counterclockwise motion around these points. For example, the vanishing $\operatorname{arcs}$ near $q_{2}$ look identical to those near $q_{1}$ except that they connect $\tilde{\alpha}_{j}$ to $\tilde{q}_{2, j}$ by passing above the points $\widetilde{q}_{1,1}, \ldots, \widetilde{q}_{1,5}, z_{1}$.

The same argument holds for the monodromies near $q_{i}, z_{i}$ and $r_{i}$ for $i \geq 3$; hence, we have now determined 180 vanishing arcs for $\Delta_{\infty} \cup C_{2}$ (10 at each $q_{i}$ and 20 at each $r_{i}$ ), and 8 vanishing cycles of $f$; see Figure 8 . Using Proposition 2.8, these calculations yield 196 vanishing cycles for the pencil $\tilde{f}_{2}=f \circ \pi_{2}$ on $X_{2}$ (one for each vanishing arc of $\Delta_{\infty} \cup C_{2}$ and two for each vanishing cycle of $f$ ).

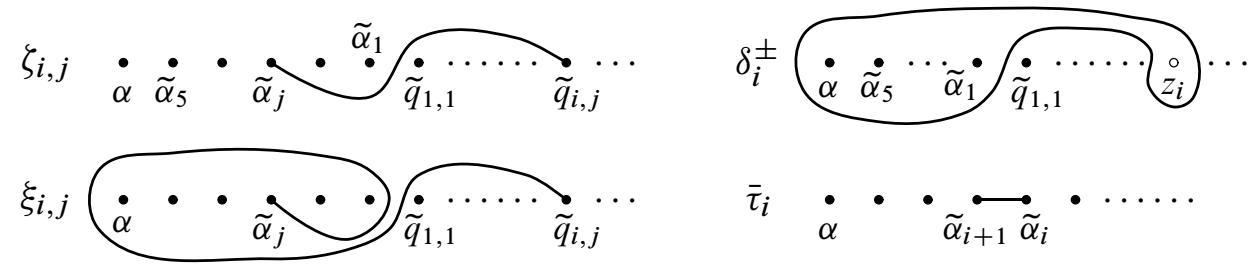

Figure 8: The vanishing cycles of the canonical pencil on $X_{2}$

\subsection{The monodromy of the canonical pencil on $X_{2}$}

Lemma 3.1 A generic curve in the linear system $\left|K_{X_{2}}\right|$ has genus 17; a generic pencil of such curves has 16 base points, and 196 nodal singularities.

Proof Since $K_{X_{2}} \cdot K_{X_{2}}=2\left[\Delta_{0}+F\right] \cdot\left[\Delta_{0}+F\right]=16$, the adjunction formula yields that a generic curve in $\left|K_{X_{2}}\right|$ has genus $g=1+K_{X_{2}} \cdot K_{X_{2}}=17$, and two such curves intersect in 16 points. By blowing up the 16 base points of a canonical pencil on $X_{2}$, we 
obtain a surface $\hat{X}_{2}$ with Euler characteristic $e\left(\hat{X}_{2}\right)=e\left(X_{2}\right)+16=132$. This surface carries a Lefschetz fibration of genus $g=17$, and the Euler characteristic is related to the number $N$ of nodal singularities by the classical formula $e\left(\hat{X}_{2}\right)=4-4 g+N$, which implies that $N=196$.

Hence, the monodromy of a generic pencil of curves in the linear system $\left|K_{X_{2}}\right|$ can be expressed in terms of 196 Dehn twists in the mapping class group Map ${ }_{17,16}$; in particular, this confirms that all the vanishing cycles of the pencil $\tilde{f}_{2}=f \circ \pi_{2}$ have been accounted for in the above calculations.

Recall that we view the reference fiber $\tilde{\Sigma}$ of $\tilde{f}_{2}$ as a double cover of a sphere with 8 punctures $\Sigma=\mathbb{C P}^{1} \backslash\left\{z_{1}, \ldots, z_{8}\right\}$, branched in 36 points. Also recall that, in $\Sigma$, the punctures $\left\{z_{i}, 1 \leq i \leq 8\right\}$ and the branch points $\left\{\alpha, \tilde{\alpha}_{j}, \tilde{q}_{i, j}, 1 \leq i \leq 6,1 \leq j \leq 5\right\}$ all lie on the real axis, in the order

$$
\begin{array}{r}
\alpha<\tilde{\alpha}_{5}<\cdots<\tilde{\alpha}_{1}<\tilde{q}_{1,1}<\cdots<\widetilde{q}_{1,5}<z_{1}<\tilde{q}_{2,1}<\cdots<\tilde{q}_{2,5}<z_{2}<\ldots \\
\cdots<z_{5}<\widetilde{q}_{6,1}<\cdots<\tilde{q}_{6,5}<z_{6}<z_{7}<z_{8} . \\
\end{array}
$$

With this notation, the calculations in Section 3.3 imply:

Theorem 3.2 Up to global conjugation and Hurwitz equivalence, the monodromy of a generic pencil of curves in the linear system $\left|K_{X_{2}}\right|$ is expressed by the factorization of the boundary twist into the following product of 196 Dehn twists:

$$
\prod_{i=1}^{6}\left[\prod_{j=1}^{5} \zeta_{i, j} \cdot \prod_{j=1}^{5} \xi_{i, j} \cdot \delta_{i}^{+} \cdot \delta_{i}^{-} \cdot\left(\bar{\tau}_{1} \cdot \bar{\tau}_{2} \cdot \bar{\tau}_{3} \cdot \bar{\tau}_{4}\right)^{5}\right] \cdot \delta_{7}^{+} \cdot \delta_{7}^{-} \cdot \delta_{8}^{+} \cdot \delta_{8}^{-},
$$

where $\zeta_{i, j}, \xi_{i, j}, \delta_{i}^{ \pm}$, and $\bar{\tau}_{i}$ are the Dehn twists along the preimages of the arcs and curves in $\Sigma$ represented in Figure 8.

Remark 3.3 Our convention is to write products of elements in the mapping class group in the left-to-right order, consistently with the standard convention for braid groups. Hence, $\varphi_{1} \cdot \varphi_{2}$ is the mapping class represented by the composition $\varphi_{2} \circ \varphi_{1}$.

\section{A symplectic Lefschetz pencil on $X_{1}$}

\subsection{Horikawa surfaces and Luttinger surgery}

The Hirzebruch surface $\mathbb{F}_{6}$ can be realized as the fiber sum of the Hirzebruch surfaces $\mathbb{F}_{2}$ and $\mathbb{F}_{4}$, in such a way that $\Delta_{\infty} \cup C_{2}$ decomposes into the fiber sum of two curves 
$D_{2} \subset \mathbb{F}_{2}$ and $D_{4} \subset \mathbb{F}_{4}$. Here each $D_{k}(k \in\{2,4\})$ is the disjoint union of the exceptional section (of square $-k$ ) and a smooth curve in the linear system corresponding to five times a section of square $+k$. Hence, the genus 2 fibration $\varphi_{2}: X_{2} \rightarrow \mathbb{C P}^{1}$ introduced in Section 2.3 (obtained by composing the double cover $\pi_{2}: X_{2} \rightarrow \mathbb{F}_{6}$ with the standard projection $p r: \mathbb{F}_{6} \rightarrow \mathbb{C P}^{1}$ ) is actually the fiber sum of two genus 2 fibrations similarly defined on the double covers of $\mathbb{F}_{2}$ and $\mathbb{F}_{4}$ branched along $D_{2}$ and $D_{4}$.

With the notations of Section 3.1, let $\gamma$ be a loop in $\mathbb{C P}^{1} \backslash \operatorname{crit}\left(\varphi_{2}\right)$ which bounds a disc $\mathrm{D}_{-} \subset \mathbb{C P}^{1}$ containing the points $q_{1}, z_{1}, r_{1}, q_{2}, z_{2}, r_{2}$, while the points $q_{i}, z_{i}, r_{i}$ for $i \geq 3$ lie in $\mathrm{D}_{+}=\mathbb{C P}^{1} \backslash \mathrm{D}_{-}$. The vertical tangencies of $\Delta_{\infty} \cup C_{2}$ lie near the points $r_{i}, 1 \leq i \leq 6$, and each $r_{i}$ contributes $\left(\sigma_{1} \cdot \sigma_{2} \cdot \sigma_{3} \cdot \sigma_{4}\right)^{5}$ to the braid monodromy factorization. Hence, the fiber sum decomposition described above corresponds exactly to a decomposition of $\mathbb{C P} P^{1}$ into the two discs $\mathrm{D}_{ \pm}$. The braid monodromy of $\Delta_{\infty} \cup C_{2}$ over D - can be represented by the factorization $\left(\sigma_{1} \cdot \sigma_{2} \cdot \sigma_{3} \cdot \sigma_{4}\right)^{10}$ in $B_{6}\left(S^{2}\right)$, while the braid monodromy over $\mathrm{D}_{+}$is $\left(\sigma_{1} \cdot \sigma_{2} \cdot \sigma_{3} \cdot \sigma_{4}\right)^{20}$; these two expressions are precisely the braid monodromy factorizations of $D_{2}$ and $D_{4}$ with respect to the natural projections. A similar property holds for the monodromies of the corresponding genus 2 Lefschetz fibrations.

The braid monodromy of $\Delta_{\infty} \cup C_{2}$ along $\gamma$ is trivial, and hence preserves any arc in the fiber of $\mathbb{F}_{6}$ with end points on the branch curve. This allows us to apply the considerations of Section 2.4. Namely, choose the reference fiber of $\mathbb{F}_{6}$ to be the fiber above a point of $\gamma$, and in this fiber let $\eta$ be the supporting arc of the standard half-twist $\sigma_{5} \in B_{6}\left(S^{2}\right)$, so that one end point of $\eta$ lies on $C_{2}$ and the other lies on $\Delta_{\infty}$; we additionally assume that $\eta$ lies away from the point where the zero section hits the reference fiber. In fact, any other arc in the reference fiber with one end point on $C_{2}$ and the other in $\Delta_{\infty}$ and avoiding the zero section would be equally suitable.

As in Section 2.4, consider the annulus $A=\gamma \times \eta \subset \mathbb{F}_{6}$ (with one boundary on $\Delta_{\infty}$ and the other on $C_{2}$ ), and its preimage $T=\pi_{2}^{-1}(A)$, which is a smoothly embedded torus in $X_{2}$. Up to suitable perturbations, $A$ and $T$ can be assumed to be Lagrangian.

Let $C^{\prime} \subset \mathbb{F}_{6}$ be the symplectic curve obtained by braiding $\Delta_{\infty} \cup C_{2}$ along the annulus $A$. This curve is connected, coincides with $\Delta_{\infty} \cup C_{2}$ outside of a neighborhood of $A$, intersects the fibers of $\mathbb{F}_{6}$ transversely in a neighborhood of $A$, and represents the same homology class $5\left[\Delta_{0}\right]+\left[\Delta_{\infty}\right]$ as $\Delta_{\infty} \cup C_{2}$. Recall that we denote by $C_{1}$ a smooth algebraic curve of bidegree $(6,12)$ in $\mathbb{C P}^{1} \times \mathbb{C P}^{1}$ (the branch curve of $\left.\pi_{1}: X_{1} \rightarrow \mathbb{C P}^{1} \times \mathbb{C P}^{1}\right)$.

Proposition 4.1 Equip $\mathbb{F}_{6}$ and $\mathbb{C P} \mathbb{P}^{1} \times \mathbb{C P}^{1}$ with fixed Kähler forms in the cohomology classes Poincaré dual to $\left[\Delta_{0}\right]+[F]$ and $(1,4)$, respectively. Then there exists a symplectomorphism $\psi: \mathbb{C P}^{1} \times \mathbb{C P}^{1} \rightarrow \mathbb{F}_{6}$ such that $\psi\left(C_{1}\right)=C^{\prime}$. 
Proof It is a classical fact that $\mathbb{F}_{6}$ and $\mathbb{C P}^{1} \times \mathbb{C P}^{1}$ are diffeomorphic; such a diffeomorphism can be assumed to preserve the fiber class, and map the homology classes $\left[\Delta_{0}\right]$ and $\left[\Delta_{\infty}\right]$ to $(1,3)$ and $(1,-3)$ respectively. Moreover, it is well-known that $\mathbb{F}_{6}$ and $\mathbb{C P}^{1} \times \mathbb{C P}^{1}$ equipped with the chosen Kähler forms are in fact symplectomorphic, even though their complex structures are different (see eg [19, Section 9.4]; see also [18]).

Hence, we can think of $C^{\prime}$ as a connected symplectic curve in $\mathbb{C P}^{1} \times \mathbb{C P} \mathbb{P}^{1}$, representing the homology class $5 \cdot(1,3)+(1,-3)=(6,12)$. We now appeal to the following isotopy result, due to Siebert and Tian [24]:

Theorem 4.2 (Siebert-Tian) Let $\Sigma \subset \mathbb{C P}^{1} \times \mathbb{C P}^{1}$ be a connected symplectic submanifold, such that the intersection number between $\Sigma$ and the fiber class is at most 7 . Then $\Sigma$ is symplectically isotopic to a holomorphic curve.

Since any two smooth holomorphic curves in the homology class $(6,12)$ are mutually isotopic, we conclude that $C^{\prime}$ is symplectically isotopic to the branch curve $C_{1}$ of $\pi_{1}: X_{1} \rightarrow \mathbb{C P}^{1} \times \mathbb{C P}^{1}$. This implies the existence of a symplectomorphism of $\mathbb{C P}^{1} \times$ $\mathbb{C P}^{1}$ (in fact, an isotopy, see eg [24, Proposition 0.2]) which maps $C_{1}$ to $C^{\prime}$.

Theorem 4.3 The manifold obtained from $\left(X_{2}, \omega_{2}\right)$ by Luttinger surgery along the torus $T$ in the direction of $\pi_{2}^{-1}(\eta)$ is symplectomorphic to $\left(X_{1}, \omega_{1}\right)$.

Proof As we have seen in Section 2.4, the symplectic manifold $\left(X^{\prime}, \omega^{\prime}\right)$ obtained from $\left(X_{2}, \omega_{2}\right)$ by Luttinger surgery along $T$ is precisely the double cover of $\mathbb{F}_{6}$ branched along $C^{\prime}$. In fact, recall that the double cover of a symplectic 4-manifold branched along a symplectic curve carries a natural symplectic structure, canonically determined up to symplectomorphism (see eg [6, Proposition 3.2]). The symplectic forms $\omega^{\prime}$ and $\omega_{1}$ are precisely those induced on the double covers $X^{\prime}$ and $X_{1}$ by the chosen Kähler forms on $\mathbb{F}_{6}$ and $\mathbb{C P}^{1} \times \mathbb{C P}^{1}$. Hence, the symplectomorphism $\psi$ given by Proposition $4.1 \mathrm{can}$ be lifted to a symplectomorphism from $\left(X^{\prime}, \omega^{\prime}\right)$ to $\left(X_{1}, \omega_{1}\right)$.

Since the Luttinger surgery operation which yields $X_{1}$ from $X_{2}$ is carried out in a manner compatible with the genus 2 fibration $\varphi_{2}: X_{2} \rightarrow \mathbb{C P}^{1}$, it yields a symplectic Lefschetz fibration $\varphi^{\prime}: X^{\prime}=X_{1} \rightarrow \mathbb{C P}^{1}$, whose monodromy differs from that of $\varphi_{2}$ in the manner described by Proposition 2.13. Together with Proposition 2.9, this implies that the monodromy of $\varphi^{\prime}$ is described by the factorization $\left(\tau_{1} \cdot \tau_{2} \cdot \tau_{3} \cdot \tau^{\prime}\right)^{10} \cdot\left(\tau_{1} \cdot \tau_{2}\right.$. $\left.\tau_{3} \cdot \tau_{4}\right)^{20}=1$ in Map 2 , where $\tau^{\prime}=\tau_{5} \tau_{4} \tau_{5}^{-1}$. With some work, one can verify that this factorization is Hurwitz equivalent to the monodromy of $\varphi_{1}$, which means that the Lefschetz fibrations $\varphi^{\prime}$ and $\varphi_{1}$ are isomorphic. However, such a result also follows more directly from the work of Siebert and Tian [24]. 


\subsection{A symplectic Lefschetz pencil on $X_{1}$}

The monodromy of a canonical pencil on $X_{1}$ can be determined directly by the same methods as in Section 3. However this calculation would yield an expression that looks very different from that of Theorem 3.2, much like the two monodromy factorization given in Proposition 2.9 look very different, and comparing the two canonical pencils would be very difficult. On the other hand, if we can place the Lagrangian torus $T \subset X_{2}$ in standard position with respect to the canonical pencil studied in Section 3, then Theorem 4.3 and Proposition 2.13 allow us to determine immediately the monodromy of a particular symplectic Lefschetz pencil on $X_{1}$. We will then prove in Section 5 that this symplectic Lefschetz pencil is isotopic to a generic pencil of holomorphic curves in the canonical linear system. For now, our main result is the following:

Theorem 4.4 $X_{1}$ carries a symplectic Lefschetz pencil $\tilde{f}^{\prime}$ whose fibers represent the canonical class and whose monodromy is described by the factorization

$$
\begin{aligned}
\prod_{i=1}^{2}\left[\prod_{j=1}^{5} \zeta_{i, j}^{\prime} \cdot \prod_{j=1}^{5} \xi_{i, j}^{\prime} \cdot \delta_{i}^{+} \cdot \delta_{i}^{-} \cdot\left(\bar{\tau}_{1} \cdot \bar{\tau}_{2} \cdot \bar{\tau}_{3} \cdot \bar{\tau}^{\prime}\right)^{5}\right] \\
\cdot \prod_{i=3}^{6}\left[\prod_{j=1}^{5} \zeta_{i, j} \cdot \prod_{j=1}^{5} \xi_{i, j} \cdot \delta_{i}^{+} \cdot \delta_{i}^{-} \cdot\left(\bar{\tau}_{1} \cdot \bar{\tau}_{2} \cdot \bar{\tau}_{3} \cdot \bar{\tau}_{4}\right)^{5}\right] \cdot \delta_{7}^{+} \cdot \delta_{7}^{-} \cdot \delta_{8}^{+} \cdot \delta_{8}^{-}
\end{aligned}
$$

in $\operatorname{Map}_{17,16}$, where $\zeta_{i, j}, \xi_{i, j}, \delta_{i}^{ \pm}$, and $\bar{\tau}_{i}$ are the Dehn twists along the preimages of the arcs and curves represented in Figure 8 , and $\zeta_{i, j}^{\prime}=\phi \zeta_{i, j} \phi^{-1}, \xi_{i, j}^{\prime}=\phi \xi_{i, j} \phi^{-1}$, $\bar{\tau}^{\prime}=\phi \bar{\tau}_{4} \phi^{-1}$, where $\phi$ is the Dehn twist along the preimage of the line segment joining the two leftmost branch points $\alpha$ and $\tilde{\alpha}_{5}$.

Proof We consider the loop $\gamma \subset \mathbb{C P}^{1}$ and the annulus $A$ introduced in Section 4.1. Since by construction $A$ lies away from the zero section of $\mathbb{F}_{6}$, in a neighborhood of $A$ the pencil $f: \mathbb{F}_{6} \backslash\left\{p_{1}, \ldots, p_{8}\right\} \rightarrow \mathbb{C P}^{1}$ introduced in Section 3.1 is very close to the standard projection $p r: \mathbb{F}_{6} \rightarrow \mathbb{C P}^{1}$. In particular, even though $f$ does not map $A$ to $\gamma$, there is a nearby annulus $A^{\prime} \subset \mathbb{F}_{6}$ with boundary on $\Delta_{\infty} \cup C_{2}$ and with the property that $f\left(A^{\prime}\right)=\gamma$. Up to a small exact perturbation of the symplectic form we can assume that $A^{\prime}$ is Lagrangian.

Recall that by construction the loop $\gamma$ bounds a disc $D_{-}$containing the points $q_{1}, z_{1}, r_{1}, q_{2}, z_{2}, r_{2}$. Hence, the monodromy of $f$ along $\gamma$ (as an element of Map $\mathrm{M}_{0,8}$ ) is the product of two boundary twists (at the critical values $z_{1}$ and $z_{2}$ ), while the braid monodromy of $\Delta_{\infty} \cup C_{2}$ along $\gamma$ is the product of the contributions from the points inside $D_{-}$; by the calculations in Section 3.3, this is the braid which moves the points 
$\alpha, \widetilde{\alpha}_{5}, \ldots, \widetilde{\alpha}_{1}$ counterclockwise around the points $\widetilde{q}_{1,1}, \ldots, \widetilde{q}_{1,5}, z_{1}, \widetilde{q}_{2,1}, \ldots, \widetilde{q}_{2,5}, z_{2}$ by 360 degrees. The annulus $A^{\prime}$ intersects the fiber of $f$ above any point of $\gamma$ in an $\operatorname{arc} \eta^{\prime}$ which is isotopic to a straight line segment joining the two points of $\Delta_{\infty} \cup C_{2}$ labelled $\alpha$ and $\tilde{\alpha}_{5}$; as expected, the monodromy along $\gamma$ preserves the arc $\eta^{\prime}$.

Braiding $\Delta_{\infty} \cup C_{2}$ along the annulus $A^{\prime}$ yields a symplectic curve in $\mathbb{F}_{6}$ which is a small isotopic perturbation of the curve $C^{\prime}$ considered in Section 4.1; in fact, for all practical purposes we can assume that this is the same curve (for example Proposition 4.1 clearly still holds), and so we again denote it by $C^{\prime}$. By the argument in Section 2.4, the double cover $X^{\prime}$ of $\mathbb{F}_{6}$ branched along the curve $C^{\prime}$ comes equipped with a symplectic Lefschetz pencil $\tilde{f}^{\prime}$ with fibers of genus 17 , obtained as a partial twisting (along the torus $T^{\prime}=\pi_{2}^{-1}\left(A^{\prime}\right)$ ) of the pencil $\widetilde{f}_{2}=f \circ \pi_{2}$ described in Section 3 .

By Proposition 2.13, the monodromy of $\tilde{f}^{\prime}$ is obtained from that of $\tilde{f}_{2}$ by conjugating the monodromy around each critical value inside the disc D_ by the Dehn twist along the preimage of the arc $\eta^{\prime}$. (Strictly speaking, we have to assume that the loop $\gamma \subset \mathbb{C P}^{1}$ has been chosen in such a way that the base point used for monodromy calculations in Section 3 lies in $D_{+}$and close to its boundary; however, it is easy to ensure that this is the case). The factors that need to be conjugated in the expression of Theorem 3.2 are those corresponding to the monodromy near the points $q_{1}, z_{1}, r_{1}, q_{2}, r_{2}, z_{2}$, namely the first 64 factors (those corresponding to $i=1$ or $i=2$ in the product), and the conjugating Dehn twist is precisely $\phi$. Hence, we obtain the expression given in the statement of Theorem 4.4.

To complete the argument, we only need to show that the pencil $\tilde{f}^{\prime}$ on $X^{\prime}$ can be viewed as a symplectic Lefschetz pencil on $X_{1}$ whose fibers represent the canonical class. Indeed, by Proposition 4.1 there exists a symplectomorphism $\psi: \mathbb{C P}^{1} \times \mathbb{C P}^{1} \rightarrow \mathbb{F}_{6}$ such that $\psi\left(C_{1}\right)=C^{\prime}$. Composing the pencil $f: \mathbb{F}_{6} \backslash\left\{p_{1}, \ldots, p_{8}\right\} \rightarrow \mathbb{C P}^{1}$ with $\psi$, we obtain a symplectic Lefschetz pencil on $\mathbb{C P}^{1} \times \mathbb{C P}^{1}$, whose fibers are symplectic curves representing the homology class $(1,4)$. Moreover, the fibers of $f \circ \psi$ intersect $C_{1}$ transversely and positively except at isolated nondegenerate tangency points. By construction, the symplectic Lefschetz pencil $f \circ \psi \circ \pi_{1}$ on $X_{1}$ is isomorphic to the symplectic Lefschetz pencil $\tilde{f}^{\prime}$ on $X^{\prime}$ (the isomorphism is given by the symplectomorphism from $X_{1}$ to $X^{\prime}$ obtained by lifting $\psi$ to the double covers); and its fibers represent the homology class $\pi_{1}^{*}(1,4)=K_{X_{1}}$.

\section{Pencils of pseudo-holomorphic spheres in $\mathbb{C P}^{1} \times \mathbb{C P}^{1}$}

Our goal in this section is to compare the symplectic Lefschetz pencil $\tilde{f}^{\prime}$ described in Theorem 4.4 with a generic pencil $\tilde{f}_{1}$ of holomorphic curves in the linear system $\left|K_{X_{1}}\right|$. We claim: 
Theorem 5.1 The Lefschetz pencils $\tilde{f}^{\prime}$ and $\tilde{f}_{1}$ are isomorphic.

Recall from Section 4 that the Lefschetz pencil $\tilde{f}^{\prime}$ is constructed as follows. Consider the curve $C^{\prime} \subset \mathbb{F}_{6}$ obtained by twisting $\Delta_{\infty} \cup C_{2}$ along the Lagrangian annulus $A^{\prime}$, and a pencil $f: \mathbb{F}_{6} \backslash\left\{p_{1}, \ldots, p_{8}\right\} \rightarrow \mathbb{C P}^{1}$ of curves in the linear system $\left|\Delta_{0}+F\right|$. The symplectic curve $C^{\prime}$ intersects the fibers of $f$ positively and transversely except at isolated nondegenerate tangency points (which all lie away from $A^{\prime}$ ), and the pencil $\tilde{f}^{\prime}$ is obtained by lifting $f$ via the double cover $\pi^{\prime}: X_{1} \simeq X^{\prime} \rightarrow \mathbb{F}_{6}$ branched along $C^{\prime}$. Since $\mathbb{C P}^{1} \times \mathbb{C P}^{1}$ and $\mathbb{F}_{6}$ (with the chosen Kähler forms in the classes $(1,4)$ and $\left.\left[\Delta_{0}\right]+[F]\right)$ are symplectomorphic, we can also view $C^{\prime}$ as a symplectic curve in $\mathbb{C P}^{1} \times \mathbb{C P}^{1}$ representing the homology class $(6,12)$, and $f$ as a symplectic Lefschetz pencil on $\mathbb{C P}^{1} \times \mathbb{C P}^{1}$ whose fibers represent the homology class $(1,4)$.

The pencil $\tilde{f}_{1}$ can also be constructed in a similar manner, by considering a pencil of algebraic curves in the class $(1,4)$ on $\mathbb{C P}^{1} \times \mathbb{C P}^{1}$ whose fibers intersect the algebraic curve $C_{1}$ transversely except at isolated nondegenerate tangency points, and lifting it via the double cover $\pi_{1}: X_{1} \rightarrow \mathbb{C P}^{1} \times \mathbb{C P}^{1}$ branched along $C_{1}$. Moreover, recall that Siebert and Tian's isotopy result (Theorem 4.2) shows the existence of a symplectic isotopy between the curves $C^{\prime}$ and $C_{1}$, ie, a continuous one-parameter family of symplectic curves $C_{t} \subset \mathbb{C P}^{1} \times \mathbb{C P}^{1}, t \in[0,1]$, such that $C_{t}$ equals $C^{\prime}$ for $t=0$ and $C_{1}$ for $t=1$. With this understood, Theorem 5.1 is an immediate corollary of the following statement:

Proposition 5.2 There exists a continuous family of symplectic Lefschetz pencils $f_{t}$, $t \in[0,1]$ on $\mathbb{C P}^{1} \times \mathbb{C P}^{1}$ such that $f_{0}=f, f_{1}$ is a pencil of algebraic curves, and for all $t$ the curve $C_{t}$ intersects the fibers of $f_{t}$ positively and transversely except at isolated nondegenerate tangency points which lie in distinct smooth fibers of $f_{t}$.

In fact, we will equip $\mathbb{C P}^{1} \times \mathbb{C P}^{1}$ with a family of almost-complex structures $J_{t}$, $t \in[0,1]$, tamed by the fixed symplectic form $\omega$, and work with pseudoholomorphic curves. We start with:

Lemma 5.3 $\mathbb{F}_{6} \simeq \mathbb{C P}^{1} \times \mathbb{C P}^{1}$ carries an almost-complex structure $J_{0}$ tamed by $\omega$ and such that the curve $C^{\prime}$ and the fibers of the pencil $f$ are $J_{0}$-holomorphic.

Proof By construction the fibers of $f$ are holomorphic with respect to the standard complex structure $\mathbb{V}$ on $\mathbb{F}_{6}$, and so is the curve $C^{\prime}$ outside of a neighborhood of the annulus $A^{\prime}$. Hence we only need to modify $\rrbracket$ in a neighborhood of $A^{\prime}$ in order to make $C^{\prime}$ pseudoholomorphic. 
Over a neighborhood $U$ of $A^{\prime}$ (in which $C^{\prime}$ is transverse to the fibers of $f$, and outside of which $C^{\prime}$ coincides with the holomorphic curve $\Delta_{\infty} \cup C_{2}$ ), we can decompose the tangent bundle to $\mathbb{F}_{6}$ into a direct sum $T_{1} \oplus T_{2}$, where $T_{1}$ is the tangent space to the fiber of $f$ and $T_{2}$ is its symplectic orthogonal. This splitting is preserved by $\rrbracket$, and choosing orthonormal bases of $T_{1}$ and $T_{2}$ for the metric induced by $\mathbb{J}$ and $\omega$, we have

$$
\mathbb{J}=\left(\begin{array}{cc}
j_{0} & 0 \\
0 & j_{0}
\end{array}\right), \quad \text { where } j_{0}=\left(\begin{array}{rr}
0 & -1 \\
1 & 0
\end{array}\right) .
$$

At any point $p \in C^{\prime} \cap U$, the transversality of $C^{\prime}$ to the fibers of $f$ implies that we can view the tangent space $T_{p} C^{\prime}$ as the graph of a linear map $h: T_{2} \rightarrow T_{1}$; the fact that $C^{\prime}$ is a symplectic curve means that $\operatorname{det}(h)>-1$ (with respect to the area forms induced by $\omega$ on $T_{1}$ and $T_{2}$ ). The almost-complex structure

$$
J=\left(\begin{array}{ll}
1 & h \\
0 & 1
\end{array}\right)\left(\begin{array}{cc}
j_{0} & 0 \\
0 & j_{0}
\end{array}\right)\left(\begin{array}{ll}
1 & h \\
0 & 1
\end{array}\right)^{-1}=\left(\begin{array}{cc}
j_{0} & h j_{0}-j_{0} h \\
0 & j_{0}
\end{array}\right)
$$

preserves $T_{1}$ and $T_{p} C^{\prime}$. Given a vector $(X, Y) \in T_{1} \oplus T_{2}$, we have

$$
\begin{aligned}
\omega((X, Y), J(X, Y)) & =\omega\left((X, Y),\left(j_{0} X+\left(h j_{0}-j_{0} h\right) Y, j_{0} Y\right)\right) \\
& =\langle X, X\rangle-\left\langle X,\left(j_{0} h j_{0}+h\right) Y\right\rangle+\langle Y, Y\rangle,
\end{aligned}
$$

where $\langle\cdot, \cdot\rangle$ is the metric induced by $\rrbracket$ and $\omega$. However, decomposing $h$ into its complex linear and antilinear parts $h^{1,0}$ and $h^{0,1}$ (with respect to $j_{0}$ ), we have $\operatorname{det}(h)=$ $\left\|h^{1,0}\right\|^{2}-\left\|h^{0,1}\right\|^{2}$, so the norm of $\left(j_{0} h j_{0}+h\right) Y=2 h^{0,1} Y$ is less than twice the norm of $Y$. This implies that $J$ is tamed by $\omega$.

We have therefore obtained an almost-complex structure with the desired properties at every point of $C^{\prime} \cap U$; moreover, near the boundary of $U$ the curve $C^{\prime}$ is $\rrbracket_{-}$ holomorphic and hence $h$ is complex linear, so that $J$ coincides with $\rrbracket$. We can extend this construction to a tubular neighborhood of $C^{\prime} \cap U$ by choosing a suitable extension of $h$ (preserving the condition $\operatorname{det}(h)>-1$ and the complex linearity near the boundary

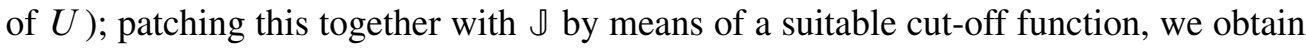
a globally defined almost-complex structure with the desired properties.

Starting from the $J_{0}$-holomorphic curve $C^{\prime}$, the method used by Siebert and Tian to prove symplectic isotopy [24] yields a family of $\omega$-tame almost-complex structures $J_{t}$, $t \in[0,1]$ on $\mathbb{C P}^{1} \times \mathbb{C P}^{1}$, with $J_{1}$ equal to the standard (product) complex structure, and a family of smooth $J_{t}$-holomorphic curves $C_{t}$ realizing the isotopy between $C^{\prime}$ and $C_{1}$.

At this point, we need to review some standard results about pseudoholomorphic spheres in $\mathbb{C P}^{1} \times \mathbb{C P}^{1}$. As observed by Hofer-Lizan-Sikorav [15], the linearized $\bar{\partial}$-operator 
is always surjective for embedded pseudoholomorphic spheres of self-intersection number at least -1 in an almost-complex 4-manifold (see also [19, Lemma 3.3.3]); this property is sometimes called automatic regularity. Hence, the moduli spaces of $J_{t}$-holomorphic spheres (ie, embedded irreducible $J_{t}$-holomorphic curves of genus 0 ) considered below are always smooth manifolds of the expected dimensions (provided they are non-empty). This implies:

Lemma 5.4 Let $J$ be any $\omega$-tame almost-complex structure on $\mathbb{C P}^{1} \times \mathbb{C P}^{1}$.

(i) Any point $p \in \mathbb{C P} \mathbb{P}^{1} \times \mathbb{C P}^{1}$ lies on a unique $J$-holomorphic sphere representing the homology class $(0,1)$, which we call the $J$-fiber through $p$.

(ii) Given an integer $k \geq 0$ and $2 k+1$ distinct points $p_{1}, \ldots, p_{2 k+1}$ in $\mathbb{C P}^{1} \times \mathbb{C P}^{1}$, there exists at most one $J$-holomorphic sphere representing the homology class $(1, k)$ and passing through the points $p_{1}, \ldots, p_{2 k+1}$.

(iii) Fix $k \geq 0$, and assume that $\mathbb{C P}^{1} \times \mathbb{C} \mathbb{P}^{1}$ contains no $J$-holomorphic spheres in the homology class $(1, j)$ for any $j<-k$. Let $p_{1}, \ldots, p_{2 k+1}$ be distinct points in $\mathbb{C P}^{1} \times \mathbb{C P}^{1}$, such that no two of them lie in the same $J$-fiber. Assume moreover that for all $-k \leq j<k$, no $j+k+1$ of the points $p_{1}, \ldots, p_{2 k+1}$ lie on the same $J$-holomorphic sphere in the homology class $(1, j)$. Then there exists a unique $J-$ holomorphic sphere representing the homology class $(1, k)$ and passing through the points $p_{1}, \ldots, p_{2 k+1}$.

Proof The first statement is classical and due to Gromov [14, Theorem 0.2.A]. The second statement is also classical and follows from positivity of intersections: if two $J$-holomorphic curves in the class $(1, k)$ intersect in $2 k+1$ distinct points then they must share a component; since we assume irreducibility, they must be equal.

To prove the third statement, we use the fact that the Gromov-Witten invariant which counts pseudoholomorphic curves of genus 0 in the class $(1, k)$ passing through $2 k+1$ points is non-zero. Indeed, when $J$ is the standard complex structure and $p_{1}, \ldots, p_{2 k+1}$ are generic, the $2 k+1$ incidence conditions determine a one-dimensional linear subspace in the vector space $H^{0}\left(\mathcal{O}_{\mathbb{P}^{1} \times \mathbb{P}^{1}}(1, k)\right)$, ie, there is a unique algebraic curve through the given points. For a generic choice of the points this curve is smooth and hence automatically regular; since the complex structure is integrable, its contribution to the Gromov-Witten invariant is 1 .

Returning to the case of arbitrary $J$, this implies the existence of a (possibly singular) $J$-holomorphic curve of genus 0 through $p_{1}, \ldots, p_{2 k+1}$ in the homology class $(1, k)$. We claim that the assumptions on $p_{1}, \ldots, p_{2 k+1}$ imply smoothness. Indeed, if the curve is not smooth then it must be reducible and a union of smoothly embedded 
$J$-holomorphic spheres (this follows eg from the adjunction formula). However, by positivity of intersection with the $J$-fibers, every irreducible $J$-holomorphic curve must represent a homology class of the form $(a, b)$ with $a \geq 0$; and if $a=0$ then necessarily $b=1$ (positivity of area implies $b \geq 1$, and adjunction implies $b=1$ ). Therefore, our curve must be the union of a $J$-holomorphic sphere representing the homology class $(1, j)$ for some integer $-k \leq j<k$, and $k-j$ fibers. However, by assumption each of the $k-j$ fibers contains at most one of the points $p_{1}, \ldots, p_{2 k+1}$, and the component representing the class $(1, j)$ passes through at most $j+k$ of them. This yields a contradiction.

Definition 5.5 We say that a configuration of 8 distinct points $p_{1}, \ldots, p_{8} \in \mathbb{C P} \mathbb{P}^{1} \times \mathbb{C P} \mathbb{P}^{1}$ is $J$-regular if the following conditions hold: none of them lie on a $J$-holomorphic sphere in the homology class $(1, j)$ for some $j<0$; no two of them lie in a same $J$-fiber; and for all $j \in\{0,1,2,3\}$, no $2 j+2$ of them lie on a same $J$-holomorphic sphere in the homology class $(1, j)$.

Lemma 5.6 For any $\omega$-tame almost-complex structure, the set of $J$-regular configurations is a connected open subset of $\left(\mathbb{C P} P^{1} \times \mathbb{C P}^{1}\right)^{8}$ whose complement has real codimension 2 .

Proof By positivity of intersections, there is at most one $J$-holomorphic sphere representing a homology class of the form $(1, j)$ with $j<0$; configurations containing a point on such a sphere therefore form a codimension 2 subset. The moduli space of $J$-fibers has real dimension 2, so the space of configurations of two points on a same $J$-fiber has real dimension 6 , and the space of configurations of 8 points of which two lie on a same $J$-fiber has real dimension 30, ie, codimension 2. Similarly, for $0 \leq j \leq 3$, automatic regularity implies that the moduli space of $J$-holomorphic spheres in the class $(1, j)$ has real dimension $4 j+2$, the space of configurations of $2 j+2$ points on such a sphere has real dimension $8 j+6$, and the space of configurations of 8 points of which $2 j+2$ lie on such a sphere again has codimension 2 .

Proposition 5.7 Let $\left(p_{1}, \ldots, p_{8}\right)$ be a $J$-regular configuration of points in $\mathbb{C P}^{1} \times$ $\mathbb{C P}^{1}$. Then the family of all $J$-holomorphic curves of genus 0 which represent the homology class $(1,4)$ and pass through $p_{1}, \ldots, p_{8}$ forms a Lefschetz pencil. Moreover, the 8 singular fibers of this pencil are reducible $J$-holomorphic curves consisting of the $J$-fiber through some $p_{i}(1 \leq i \leq 8)$ and the $J$-holomorphic sphere in the class $(1,3)$ through all $p_{j}, j \neq i$

Proof Since $[\omega]$ is Poincare dual to $(1,4)$, there are no symplectic spheres in the homology classes $(1, j)$ for $j \leq-4$. Hence, Lemma 5.4 (iii) implies the existence 
of a unique $J$-holomorphic sphere in the homology class $(1,3)$ through any 7 of the points $p_{1}, \ldots, p_{8}$.

Consider any point $p \in \mathbb{C P}^{1} \times \mathbb{C P}^{1} \backslash\left\{p_{1}, \ldots, p_{8}\right\}$. There are two cases. If $p$ does not lie on any of the $J$-fibers through $p_{1}, \ldots, p_{8}$, nor on any of the $J$-holomorphic spheres in the homology class $(1,3)$ through seven of these points, then one easily checks that the 9 points $p_{1}, \ldots, p_{8}, p$ satisfy the assumptions of Lemma 5.4 (iii), and so they lie on a unique $J$-holomorphic sphere in the homology class $(1,4)$. Otherwise, $p_{1}, \ldots, p_{8}, p$ lie on a reducible $J$-holomorphic curve of the type described in the statement of the proposition, and by positivity of intersections no other $J$-holomorphic curve representing the homology class $(1,4)$ can pass through these 9 points.

To see that this family of $J$-holomorphic curves is parameterized by $\mathbb{C P}^{1}$, consider a $J-$ fiber $F$ (not passing through any $p_{i}$ ), and observe that each curve in the family intersects $F$ transversely in a single point, and conversely through any point of $F$ there is a single curve in the family. Hence, we can define a map from $\mathbb{C P}^{1} \times \mathbb{C P}^{1} \backslash\left\{p_{1}, \ldots, p_{8}\right\}$ to $\mathbb{C P}^{1} \simeq F$ by mapping each point $p$ to the point where the curve through $p_{1}, \ldots, p_{8}, p$ intersects $F$. The fact that this is a Lefschetz pencil follows from automatic regularity and standard arguments about deformations of $J$-holomorphic curves; in particular, the structure of the moduli space near the nodal curves follows from gluing arguments (see eg [25, Corollary 2]). In fact, the key ingredient is again automatic regularity, which implies that the local behavior of families of $J$-holomorphic spheres is the same as in the usual holomorphic case.

Proof of Proposition 5.2 Consider the family of almost-complex structures $J_{t}$ and pseudoholomorphic curves $C_{t}$ introduced above. By Lemma 5.6, we can find a continuous family of $J_{t}$-regular configurations $\left(p_{1, t}, \ldots, p_{8, t}\right), t \in[0,1]$, starting from the configuration chosen in Section 3.1 at $t=0$ (which is easily seen to be $J_{0}-$ regular, recalling that $\Delta_{0} \subset \mathbb{F}_{6}$ represents the class $(1,3)$ in $\left.\mathbb{C P}^{1} \times \mathbb{C P}^{1}\right)$. Moreover, we can choose these points in such a way that, for all $1 \leq i \leq 8$, the point $p_{i, t}$ does not lie on $C_{t}$, and the point where the $J_{t}$-fiber through $p_{i, t}$ intersects the $J_{t}$-holomorphic sphere in the class $(1,3)$ through all $p_{j, t}, j \neq i$, does not lie on $C_{t}$ either.

By Proposition 5.7, for each $t$ the points $\left(p_{1, t}, \ldots, p_{8, t}\right)$ determine a pencil $f_{t}$ of $J_{t}$-holomorphic curves in the homology class $(1,4)$. Since the curve $C_{t}$ is irreducible, every intersection of $C_{t}$ with a fiber of $f_{t}$ has a finite positive multiplicity; see eg [19, Appendix E] for a detailed discussion of this fact, which follows from Micallef and White's result about the local structure of pseudoholomorphic curves [20]. Moreover, the restriction of $f_{t}$ to $C_{t}$ is an open mapping $\phi_{t}: C_{t} \rightarrow \mathbb{C P} \mathbb{P}^{1}$ of degree 36 , whose critical points are precisely the non-transverse intersections between $C_{t}$ and fibers of $f_{t}$. 
The preimage by $\phi_{t}$ of a small disc in $\mathbb{C P}^{1}$ centered at a critical value $z_{c r}$ of $\phi_{t}$ (chosen generically so its boundary is transverse to $\left.\phi_{t}\right)$ consists of at most $\left|\phi_{t}^{-1}\left(z_{c r}\right)\right| \leq 35$ components, each of which has Euler characteristic at most 1. Hence, arguing as in the classical Hurwitz formula for branched covers, we conclude that $\phi_{t}$ has at most 180 critical points; in particular, the points where $C_{t}$ is tangent to the fibers of $f_{t}$ are isolated.

We conclude that $\phi_{t}: C_{t} \rightarrow \mathbb{C P} \mathbb{P}^{1}$ is a topological branched covering. After modifying $\phi_{t}$ by a $C^{1}$-small perturbation supported in a neighborhood of its critical points, we can assume that the critical points of $\phi_{t}$ are all non-degenerate, and that the corresponding critical values are distinct from each other and from the critical values of $f_{t}$. Using suitable cut-off functions, this modification of $\phi_{t}$ can be extended to a $C^{1}$-small perturbation of $f_{t}$ supported in a neighborhood of the critical points of $\phi_{t}$, preserving the property that the fibers of the pencil intersect $C_{t}$ positively. The fibers of the perturbed pencil are no longer $J_{t}$-holomorphic, but they can still be assumed to be symplectic. It is moreover clear that this perturbation argument can be carried out in a manner such that the perturbations depend continuously on $t \in[0,1]$.

\section{Comparing the canonical pencils}

We now have all the necessary ingredients to compare generic pencils of curves in the canonical linear systems on $X_{1}$ and $X_{2}$. In particular, Theorem 1.2 follows directly from Theorem 3.2, Theorem 4.4, and Theorem 5.1. Moreover, in order to compare the monodromy groups and prove Theorem 1.3, it is enough to prove that the conjugating element $\phi$ belongs to the monodromy subgroups of both pencils. Namely, we have to prove:

Proposition 6.1 With the notations of Theorem 1.2, the Dehn twist $\phi$ belongs to the subgroup $G_{2}$ of $\mathrm{Map}_{17,16}$ generated by $t_{1}, \ldots, t_{196}$, and it also belongs to the subgroup $G_{1}$ generated by $\phi t_{1} \phi^{-1}, \ldots, \phi t_{64} \phi^{-1}, t_{65}, \ldots, t_{196}$. Therefore, $G_{1}=G_{2}$.

It is easy to prove that $\phi \in G_{1}$. Indeed, recall that Dehn twists along simple closed curves that intersect transversely once satisfy the relation $t_{a} t_{b} t_{a}=t_{b} t_{a} t_{b}$. Therefore, with the notations of Theorem 4.4, we have $\bar{\tau}^{\prime}=\phi \bar{\tau}_{4} \phi^{-1}=\bar{\tau}_{4}^{-1} \phi \bar{\tau}_{4}$, so $\phi=\bar{\tau}_{4} \bar{\tau}^{\prime} \bar{\tau}_{4}^{-1}$ obviously belongs to the subgroup $G_{1}$ generated by the various Dehn twists appearing in Theorem 4.4.

The argument for $X_{2}$ is more subtle, since a quick inspection of the factors in Theorem 3.2 does not suggest any obvious reason why $\phi$ should belong to the monodromy group.

Geometry $\&$ Topology, Volume 10 (2006) 
We use the same notations as in Section 3.4; in particular, we consider Dehn twists in Map $_{17,16}$ which are obtained by lifting half-twists or Dehn twists via the double cover $\pi_{2}: \widetilde{\Sigma} \rightarrow \Sigma$, where $\Sigma=\mathbb{C P}^{1} \backslash\left\{z_{1}, \ldots, z_{8}\right\}$ and the 36 branch points of $\pi_{2}$ are labelled as in Section 3.4.

Lemma 6.2 The subgroup of Map ${ }_{17,16}$ generated by the Dehn twists $\zeta_{i, j}$ and $\bar{\tau}_{j}$ is the image by the lifting homomorphism of the braid group $B_{35}$ consisting of all braids supported in a disc $\mathrm{D}_{0} \subset \Sigma$ which contains the 35 branch points $\tilde{\alpha}_{j}$ and $\tilde{q}_{i, j}$ as well as arcs connecting them within the upper half-plane, as shown on Figure 9.

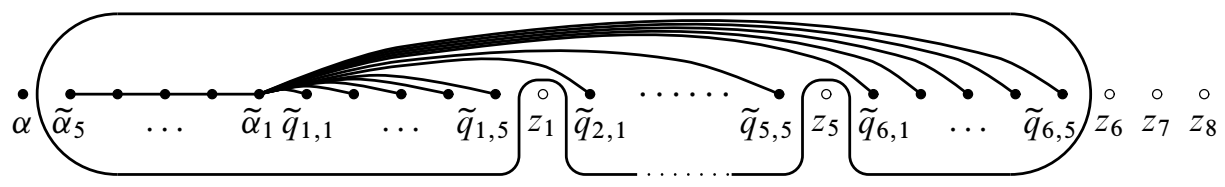

Figure 9: The disc $\mathrm{D}_{0}$ and the tree $T_{0}$

Proof Conjugating $\zeta_{i, j}$ by $\bar{\tau}_{j-1} \ldots \bar{\tau}_{1}$, we obtain the lift of a half-twist that exchanges the branch points $\widetilde{\alpha}_{1}$ and $\tilde{q}_{i, j}$ along an arc contained in the upper half-plane. These 30 arcs together with the 4 arcs supporting the half-twists which lift to $\bar{\tau}_{1}, \ldots, \bar{\tau}_{4}$ form an embedded tree $T_{0} \subset \Sigma$ (see Figure 9). It is well-known that these half-twists generate the braid group $B_{35}$ (for example, further conjugations yield half-twists whose supporting arcs form a linear chain as in Artin's standard set of generators).

Definition 6.3 The upper envelope of a subset $S$ of $S_{0}=\left\{\alpha, \widetilde{\alpha}_{j}, \widetilde{q}_{i, j}, i=1 \ldots 6\right.$, $j=1 \ldots 5\} \cup\left\{z_{i}, 1 \leq i \leq 8\right\}$ is the simple closed curve $c(S) \subset \Sigma$ which bounds a disc containing the points of $S$ as well as arcs connecting them within the upper half-plane, but not any points of $S_{0} \backslash S$. We denote by $\delta(S)^{ \pm}$the two lifts of the Dehn twist along $c(S)$.

For example, the factors $\delta_{i}^{ \pm}$in Theorem 3.2 are the lifts of the Dehn twists along the upper envelopes of the sets $\left\{\alpha, \widetilde{\alpha}_{5}, \ldots, \widetilde{\alpha}_{1}, z_{i}\right\}$. With this terminology, the following result is an immediate corollary of Lemma 6.2:

Lemma 6.4 If the monodromy group $G_{2}$ contains $\delta(S)^{ \pm}$for some set $S$, then it also contains $\delta\left(S^{\prime}\right)^{ \pm}$for any set $S^{\prime}$ which is the image of $S$ by a permutation of $S_{0}$ fixing the elements $\alpha, z_{1}, \ldots, z_{8}$.

In particular, $G_{2}$ contains $\delta(S)^{ \pm}$for any 7-element set $S$ which contains $\alpha$ and exactly one $z_{i}$. Our next observation is the following: 
Lemma 6.5 Let $c, c^{\prime}$ be two simple closed curves in $\Sigma$, intersecting in two points as in Figure 10, left, so one of the regions delimited by $c \cup c^{\prime}$ contains a single branch point of $\pi_{2}$. Assume that $G_{2}$ contains the Dehn twists along both lifts of $c$ and $c^{\prime}$. Then $G_{2}$ also contains the Dehn twists along both lifts of the loops $c_{ \pm}$obtained by "summing" $c$ and $c^{\prime}$ (Figure 10, right).

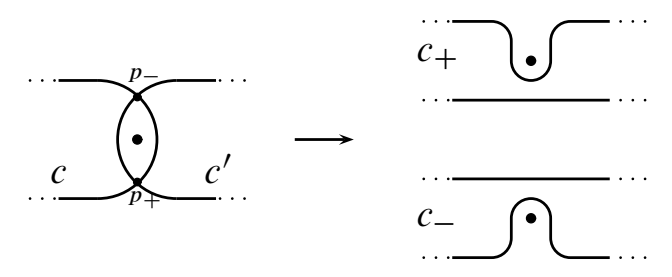

Figure 10: Summing simple closed curves

Proof Let $\tilde{c}$ and $\tilde{c}^{\prime}$ be arbitrary lifts of $c$ and $c^{\prime}$. The loops $\tilde{c}$ and $\tilde{c}^{\prime}$ intersect only once, at a point of $\pi_{2}^{-1}\left(c \cap c^{\prime}\right)$ which depends on the chosen lifts. Hence, denoting by $\tilde{t}$ and $\tilde{t}^{\prime}$ the corresponding Dehn twists, the conjugate of $\tilde{t}^{\prime}$ by $\tilde{t}$ is the Dehn twist along the connected sum of $\tilde{c}$ with $\widetilde{c}^{\prime}$. Assume that the intersection of $\tilde{c}$ with $\widetilde{c}^{\prime}$ lies above $p_{+}$: then $\widetilde{t t^{\prime}} \tilde{t}^{-1}$ is the Dehn twist along $\widetilde{t}^{-1}\left(c^{\prime}\right)$, which is a lift of $c_{+}$since the lift of a loop that encircles the branch point twice is contractible. Similarly, if the intersection of $\tilde{c}$ and $\widetilde{c}^{\prime}$ lies above $p_{-}$, then $\tilde{t}^{-1} \widetilde{t}^{\prime} \tilde{t}$ is the Dehn twist along a lift of $c_{-}$. Considering the four possible choices for $\left(\widetilde{c}, \tilde{c}^{\prime}\right)$, we obtain both lifts of $c_{+}$and both lifts of $c_{-}$.

In particular, let $S$ and $S^{\prime}$ be two subsets of $S_{0}$ such that $S \cap S^{\prime}$ consists of a single element $s$ which is not one of the $z_{i}$. Assume moreover that, with respect to the natural ordering of the elements of $S_{0}$ induced by their positions along the real axis, the following conditions are satisfied:

- $s \in\left\{\inf \left(S^{\prime}\right), \sup \left(S^{\prime}\right)\right\}$;

- $S \cap\left[\inf \left(S^{\prime}\right), \sup \left(S^{\prime}\right)\right]=\{s\}$.

In this situation, if the monodromy group $G_{2}$ contains $\delta(S)^{ \pm}$and $\delta\left(S^{\prime}\right)^{ \pm}$, then by Lemma 6.5 it also contains $\delta(\bar{S})^{ \pm}$where $\bar{S}=\left(S \cup S^{\prime}\right) \backslash\{s\}$.

Applying this argument repeatedly to specific subsets of $S_{0}$ satisfying these conditions, and combining with Lemma 6.4 , we conclude that $G_{2}$ contains $\delta(S)^{ \pm}$whenever $S$ satisfies one of the following conditions:

- $S$ contains 7 elements, among which one $z_{i}$, and $\alpha \in S$; 
- $S$ contains 12 elements, among which two $z_{i}$, and $\alpha \notin S$;

- $S$ contains 17 elements, among which three $z_{i}$, and $\alpha \in S$;

- $S$ contains 22 elements, among which four $z_{i}$, and $\alpha \notin S$;

- $S$ contains 27 elements, among which five $z_{i}$, and $\alpha \in S$;

- $S$ contains 32 elements, among which six $z_{i}$, and $\alpha \notin S$;

- $S$ contains 37 elements, among which seven $z_{i}$, and $\alpha \in S$;

- $S$ contains 42 elements, among which eight $z_{i}$, and $\alpha \notin S$.

In particular, taking $S_{\phi}=S_{0} \backslash\left\{\alpha, \tilde{\alpha}_{5}\right\}$ (which contains 42 elements, including all the $z_{i}$ ) we obtain that $\delta\left(S_{\phi}\right)^{ \pm} \in G_{2}$; however it is easy to see that $\delta\left(S_{\phi}\right)^{ \pm}=\phi$, which completes the proof of Proposition 6.1.

\section{Pluricanonical pencils and degree doubling}

As mentioned in Section 2.1, comparing Horikawa surfaces requires understanding not only pencils of curves in the canonical linear system, but also in the pluricanonical linear systems $\left|k K_{X_{i}}\right|, k \gg 1$. This can be achieved by means of a degree doubling procedure, which describes the topology of a pencil of curves in the linear system $\left|2 k K_{X_{i}}\right|$ in terms of that of a pencil in the linear system $\left|k K_{X_{i}}\right|$. This idea, which goes back to Donaldson [7], has been explored in greater detail in [26] and [3]. We start by giving an outline of the relevant material in those two papers.

Consider a Lefschetz pencil $f=\left(s_{0} / s_{1}\right)$ defined by two generic sections $s_{0}, s_{1}$ of a sufficiently positive line bundle $L^{\otimes k}$ (either holomorphic, or approximately holomorphic in the sense of Donaldson [8]). As observed in [26], the two sections $s_{0}^{2}$ and $s_{0} s_{1}$ of $L^{\otimes 2 k}$ define a (highly non-generic) pencil of reducible nodal curves $\left\{s_{0}^{2}-\alpha s_{0} s_{1}=0\right\}_{\alpha \in \mathbb{C P}^{1}}$, obtained by adding in the zero set of $s_{0}$ to each of the curves in the original pencil. A generic pencil can be obtained by choosing small perturbations $\epsilon_{0}, \epsilon_{1}$ (sections of $L^{\otimes 2 k}$ ) and considering the sections $s_{0}^{2}+\epsilon_{0}$ and $s_{0} s_{1}+\epsilon_{1}$ instead. It is then easy to see that the generic fiber of this pencil is obtained by forming the connected sum of two generic fibers of $f$ (smoothing the intersections at the base points), and that the critical points and vanishing cycles which occur away from the zero set of $s_{0}$ are in one-to-one correspondence with those of $f$ [26]. If $f$ has fiber genus $g$ and $n$ base points, then the doubled pencil has fiber genus $\bar{g}=2 g+n-1$ and $\bar{n}=4 n$ base points. Its monodromy consists of: (1) the image of the monodromy of $f$ under a natural embedding $\operatorname{Map}_{g, n} \hookrightarrow \operatorname{Map}_{\bar{g}, \bar{n}}$ induced by viewing the fiber of $f$ (minus a neighborhood of its base points) as a subset of the new fiber, and (2) contributions from a neighborhood of the zero set of $s_{0}$ [26]. 
The work in [3] aims to turn these considerations into an explicit formula for the monodromy of the doubled pencil. The starting point is that complex surfaces, and more generally symplectic 4-manifolds (see [4]), can be realized as branched covers of $\mathbb{C P}^{2}$ by choosing three suitable sections $s_{0}, s_{1}, s_{2}$ of the line bundle $L^{\otimes k}$, and considering the map $F=\left(s_{0}: s_{1}: s_{2}\right): X \rightarrow \mathbb{C P}^{2}$. Choosing the sections generically in the holomorphic case, or in a specific manner in the approximately holomorphic case, we can ensure that $F$ is a branched covering with generic local models, ie, near any point of the ramification curve $R \subset X$ it is modelled on one of the two maps $(x, y) \mapsto\left(x^{2}, y\right)$ or $(x, y) \mapsto\left(x^{3}-x y, y\right)$; moreover the branch curve $D=F(R) \subset \mathbb{C P}^{2}$ can be assumed to have the following properties:

(1) the only singularities of $D$ are ordinary double points ("nodes") and ordinary cusps (in the approximately holomorphic case, there may be some double points with the anti-complex orientation);

(2) $F_{\mid R}: R \rightarrow D$ is an immersion everywhere except at the cusps, and one-to-one except at the nodes;

(3) $(0: 0: 1) \notin D$;

(4) $D$ is positively transverse to the fibers of the linear projection $\pi:(x: y: z)$ $\mapsto(x: y)$ from $\mathbb{C P}^{2}$ to $\mathbb{C P}^{1}$, except at isolated nondegenerate tangency points (distinct from the cusps and nodes);

(5) the cusps, nodes, and tangency points lie in different fibers of $\pi$.

Then the composition $f=\pi \circ F=\left(s_{0} / s_{1}\right)$ is a Lefschetz pencil. The singular fibers of $f$ are the preimages by $F$ of those fibers of $\pi$ which are tangent to $D$, its base points are the preimages of $(0: 0: 1)$, and its monodromy can be determined from the braid monodromy of $D$ using a lifting homomorphism as in Section 2.2.

As observed in [3], the composition of $F$ with a generic quadratic map $V_{2}^{\prime}: \mathbb{C P}^{2} \rightarrow \mathbb{C P}^{2}$ yields a map $\bar{F}=V_{2}^{\prime} \circ F: X \rightarrow \mathbb{C P}^{2}$ determined by three sections of $L^{\otimes 2 k}$ which are quadratic expressions in $s_{0}, s_{1}, s_{2}$; this map is again a branched covering, ramified along $\bar{R}=R \cup F^{-1}\left(R^{\prime}\right)$ (where $R^{\prime}$ is the ramification curve of $V_{2}^{\prime}$ ). The branch curve of $\bar{F}$ consists of two parts, namely $V_{2}^{\prime}(D)$ on one hand, and $n=\operatorname{deg} F$ superimposed copies of the branch curve $D^{\prime}$ of $V_{2}^{\prime}$ on the other hand. Near a point where $F^{-1}\left(R^{\prime}\right)$ intersects $R$, a local model for $\bar{F}$ is given by the composition of two simple branched covers such that the branch curve of the first map is in general position with respect to the second one, $(x, y) \mapsto\left(x^{2}+y, y\right) \mapsto\left(x^{2}+y, y^{2}\right)$. At such a point, the branch curve of $\bar{F}$ is not immersed, and presents a self-tangency (since $V_{2}^{\prime}(D)$ is tangent to $D^{\prime}$ ). So, $\bar{F}$ is not everywhere given by one of the generic local models, and its branch curve $\bar{D}$ does not satisfy properties (1) and (2) above: in addition to nodes and 
cusps, $\bar{D}$ also has self-tangencies, and while the restriction of $\bar{F}$ to its ramification curve is still an immersion outside the cusps and self-tangencies, it is not generically one-to-one. Nonetheless, a small perturbation can be added to $\bar{F}$ in order to get a covering with generic local models, satisfing properties (1)-(5). The main idea in [3] is that the topology of this covering (in particular the braid monodromy of its branch curve) can be determined explicitly from that of $F$, using the non-generic map $\bar{F}$ as an intermediate step.

After composing with the linear projection $\pi$, we again obtain a Lefschetz pencil $\bar{f}$, whose monodromy can be determined by lifting the half-twists in the braid monodromy of the branch curve. This leads to an explicit degree doubling formula for Lefschetz pencils obtained from sections of sufficiently positive line bundles [3, Theorem 4 , Section 4.2]:

Theorem 7.1 Let $f=\pi \circ F$ be a Lefschetz pencil with fiber genus $g$ and $n$ base points, determined by two sections of $L^{\otimes k}$, where $k$ is assumed to be sufficiently large. Let $\Phi$ be a factorization of the boundary twist in $\operatorname{Map}_{g, n}$ describing the monodromy of $f$. Let $\bar{f}$ be the Lefschetz pencil obtained by the construction described above (so $\bar{f}$ is determined by two sections of $L^{\otimes 2 k}$, its fiber genus is $\bar{g}=2 g+n-1$, and it has $\bar{n}=4 n$ base points).

Then the monodromy of $\bar{f}$ can be described by the factorization $\iota(\Phi) \cdot U_{g, n}$ in $\operatorname{Map}_{\bar{g}, \bar{n}}$, where $\iota: \operatorname{Map}_{g, n} \hookrightarrow \operatorname{Map}_{\bar{g}, \bar{n}}$ is a natural embedding induced by viewing the fiber of $f$ (minus a neighborhood of its base points) as a subset of the fiber of $\bar{f}$, and $U_{g, n}$ is an explicitly determined collection of $4 g-4+7 n$ Dehn twists in Map $\bar{g}, \bar{n}$ that depends only on $g$ and $n$ (but not on $f$ ).

It is not immediately clear that this approach applies to the canonical pencils on the Horikawa surfaces $X_{1}$ and $X_{2}$. The main issue is that the canonical pencils do not satisfy the "large $k$ " requirement. In particular, it is not clear that holomorphic or approximately holomorphic perturbations with the required properties can be constructed, and it is not clear either that a Lefschetz pencil obtained by approximately holomorphic techniques would be topologically equivalent to a pencil of holomorphic curves. In fact, the linear systems $\left|K_{X_{i}}\right|$ and $\left|2 K_{X_{i}}\right|$ factor through $\mathbb{C P}^{1} \times \mathbb{C P}^{1}$ and $\mathbb{F}_{6}$, so a generic triple of holomorphic sections of the canonical bundle does not even determine a map to $\mathbb{C P}^{2}$ with generic local models.

However, the features of the maps to $\mathbb{C P}^{2}$ that naturally arise in this context are actually not an obstacle. Indeed, given a branched covering map $F: X \rightarrow \mathbb{C P}^{2}$, the critical points of $\pi \circ F$ are the points of the ramification curve where the image of $d F$ is not transverse to the fiber of $\pi$, ie, the points of $R$ which map to the vertical tangencies of $D$; in particular, among the properties listed above, only (3) and (4) really matter. 
Definition 7.2 A branched covering map $F: X \rightarrow \mathbb{C P}^{2}$ is tame if near any point of the ramification curve $R \subset X$ it is modelled on one of the three maps $(x, y) \mapsto\left(x^{2}, y\right)$, $(x, y) \mapsto\left(x^{3}-x y, y\right)$, or $(x, y) \mapsto\left(x^{2}+y, y^{2}\right)$, and moreover the branch curve $D=F(R) \subset \mathbb{C P}^{2}$ satisfies the following properties:

$\left(1^{\prime}\right)$ the only singularities of $D$ are ordinary double points, ordinary cusps, and self-tangencies;

(2') $\quad F_{\mid R}: R \rightarrow D$ is an immersion away from the cusps and self-tangencies;

(3) $(0: 0: 1) \notin D$;

(4) $D$ is positively transverse to the fibers of $\pi:(x: y: z) \mapsto(x: y)$, except at isolated nondegenerate tangency points (distinct from the cusps, nodes, and self-tangencies).

If $F$ is a tame covering, then the composition $\pi \circ F$ is still a Lefschetz pencil, althouh its critical points need not lie in distinct fibers: for example, whenever a fiber of $\pi$ is tangent to a component of $D$ over which $F_{\mid R}$ is not generically one-to-one, we get a fiber of $\pi \circ F$ with multiple nodes. With this understood, we can still consider the individual Dehn twists obtained by lifting the half-twists in the braid monodromy of $D$; in the case of a component of multiplicity $\mu$, we obtain $\mu$ different Dehn twists along disjoint simple closed curves obtained by considering appropriate lifts of the supporting arc of the half-twist.

In the holomorphic setting, mild genericity conditions ensure that the composition of two tame coverings is still a tame covering. In particular, if $F: X \rightarrow \mathbb{C P}^{2}$ is a tame covering defined by a triple of holomorphic sections of $L^{\otimes k}$ and $V_{2}^{\prime}: \mathbb{C P}^{2} \rightarrow \mathbb{C P}^{2}$ is a generic quadratic map, then $V_{2}^{\prime} \circ F$ is still a tame covering. Moreover, considering specifically the Horikawa surfaces $X_{i}(i \in\{1,2\})$, the map to $\mathbb{C P}^{2}$ defined by a generic triple of sections of the canonical bundle $K_{X_{i}}$ is the composition of the double covering $\pi_{i}$ with a generic branched covering defined by three sections of $\mathcal{O}(1,4)$ on $\mathbb{C P}^{1} \times \mathbb{C P}^{1}$ or $\mathcal{O}\left(\Delta_{0}+F\right)$ on $\mathbb{F}_{6}$; such a map is a tame covering.

Given a tame covering map $F$, one can always modify it by an arbitrarily small $C^{\infty}$ perturbation (locally holomorphic near the vertical tangencies, cusps, and selftangencies) in order to obtain a symplectic branched covering with generic local models satisfying properties (1)-(5). More precisely, the effect of such a perturbation is to replace each self-tangency by three cusps (replacing $(x, y) \mapsto\left(x^{2}+y, y^{2}\right)$ by $(x, y) \mapsto\left(x^{2}+y, y^{2}+\epsilon x\right)$ for a small nonzero $\left.\epsilon\right)$, and to separate each multiple component of $D$ into distinct copies intersecting at nodes (see [3]). However, as far as the corresponding Lefschetz pencil is concerned, the only effect of the perturbation is 
to move the critical points of $\pi \circ F$ into distinct nearby fibers; so the monodromy still consists of the same Dehn twists, independently of the chosen perturbation.

Hence, while perturbations are needed in order to study the effect of degree doubling on the braid monodromy of the branch curves, which is the method used in [3] in order to derive Theorem 7.1, they are actually irrelevant as far as pencils are concerned. In particular, in our setting the formula in Theorem 7.1 can be interpreted more directly as a relation between the monodromies of the pencils $\pi \circ F$ and $\pi \circ V_{2}^{\prime} \circ F$ (with the understanding that, when several critical points lie in a same fiber, we still consider the individual Dehn twists separately). In conclusion, we have:

Proposition 7.3 Let $F: X \rightarrow \mathbb{C P}^{2}$ be a holomorphic map from a complex surface to $\mathbb{C P}^{2}$, and assume that $F$ is a tame branched covering. Let $V_{2}^{\prime}: \mathbb{C P}^{2} \rightarrow \mathbb{C P}^{2}$ be a map defined by three generic quadratic polynomials. Then the maps $f=\pi \circ F$ and $\bar{f}=\pi \circ V_{2}^{\prime} \circ F$ are Lefschetz pencils, and their monodromies are related by the formula in Theorem 7.1.

In particular, the monodromy of a generic pencil of curves in the linear system $\left|2^{m} K_{X_{i}}\right|$ on the Horikawa surface $X_{i}(m \geq 1, i \in\{1,2\})$ consists of two ingredients:

- the image of the monodromy of the canonical pencil $\tilde{f}_{i}$ under a natural embed-

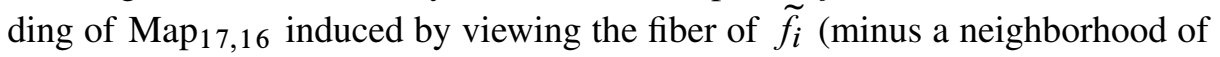
the base points) as a subset of the new fiber;

- an explicit collection of Dehn twists that depends on $m$ but not on $i$.

With this understood, Theorem 1.4 becomes an easy corollary of Theorems 1.2 and 1.3.

\section{Matching paths and Lagrangian spheres in $X_{i}$}

Lefschetz pencils can be used to understand Lagrangian spheres in a symplectic manifold via matching paths, an idea due to Donaldson and Seidel (see [7] and [22, Section 9b]). In the four-dimensional case, the definition is quite simple: 
Definition 8.1 Let $f: X^{4} \backslash B \rightarrow S^{2}$ be a symplectic Lefschetz pencil. An embedded arc $\gamma:[0,1] \rightarrow S^{2}$ with $\gamma^{-1}$ (crit $\left.f\right)=\{0,1\}$ is a matching path for $f$ if the vanishing cycles associated to the $\operatorname{arcs} \gamma\left(\left[0, \frac{1}{2}\right]\right)$ and $\gamma\left(\left[\frac{1}{2}, 1\right]\right)$ are isotopic to each other inside $f^{-1}\left(\gamma\left(\frac{1}{2}\right)\right) \backslash B$.

For example, if a same Dehn twist is repeated twice in the monodromy factorization associated to $f$, then the "simplest" arc that joins the two corresponding critical values by passing through the chosen base point is a matching path. More generally, matching paths arise whenever an arbitrary sequence of Hurwitz moves leads to a factorization in which a same Dehn twist is repeated twice.

A matching path gives rise to an embedded Lagrangian sphere in $X$ (up to isotopy), obtained by joining together the two thimbles formed by parallel transport of the vanishing cycles along the $\operatorname{arcs} \gamma\left(\left[0, \frac{1}{2}\right]\right)$ and $\gamma\left(\left[\frac{1}{2}, 1\right]\right)$; see [22, Section 9b]. Conversely, as observed by Donaldson, any Lagrangian sphere can be obtained (up to isotopy) from a matching path in a Lefschetz pencil of sufficiently high degree (see [5] for a proof).

Matching paths can be viewed as specific elements ("figure 8 loops") in the kernel of the monodromy morphism $\psi: \pi_{1}(\mathrm{D} \backslash \operatorname{crit}(f)) \rightarrow \operatorname{Map}_{g, n}$ associated to the Lefschetz pencil, or as specific pairs $\left(\gamma_{+}, \gamma_{-}\right)$of conjugates of generators of $\pi_{1}(D \backslash \operatorname{crit}(f))$ for which the monodromies coincide.

In the case of the canonical pencil $\tilde{f}_{2}$ on $X_{2}$, whose monodromy has been described in Theorem 3.2, there are obvious matching paths arising from the repeated factors $\bar{\tau}_{i}$, and slightly less obvious matching paths arising from the fact that the conjugate of $\bar{\tau}_{i}$ by $\bar{\tau}_{i+1}$ equals the conjugate of $\bar{\tau}_{i+1}$ by $\bar{\tau}_{i}^{-1}$.

The Lagrangian spheres arising from these matching paths are well understood, and correspond to the algebraic vanishing cycles mentioned in Remark 2.12, spanning the orthogonal complement to $\Lambda_{2}=\pi_{2}^{*} H^{2}\left(\mathbb{F}_{6}\right)$ in the second homology group of $X_{2}$. While it is generally expected that $X_{2}$ contains no "exotic" Lagrangian spheres (representing homology classes that are not orthogonal to $\Lambda_{2}$ ), the calculations in Section 6 give an indication of how one might start looking for unexpected matching paths in $\widetilde{f}_{2}$.

For example, since the Dehn twist $\phi$ belongs to the subgroup generated by the $\zeta_{i, j}, \delta_{i}^{ \pm}$, and $\bar{\tau}_{i}$, one could try to use the observation that $\zeta_{i, j}$ coincides with the conjugate of $\xi_{i, j}$ by $\left(\bar{\tau}_{1} \bar{\tau}_{2} \bar{\tau}_{3} \bar{\tau}_{4} \phi\right)^{6}$ as a starting point to build a matching path. Various other tricks of a similar nature come to mind (all using the Dehn twist $\phi$ to build unexpected relations among monodromy factors). However, our first naive attempts in this direction have all led to paths that are only immersed rather than embedded, and hence to immersed (rather than embedded) Lagrangian spheres. The existence of such immersed spheres is 
not very surprising if one remembers that Gromov's h-principle applies to Lagrangian immersions. In fact, the difference between immersed and embedded objects is a recurring theme in 4-manifold topology, and it is interesting to see it appear in this situation.

Similar considerations come up when investigating matching paths in the canonical pencil $\tilde{f}_{1}$ on $X_{1}$. In both cases, this suggests the following directions for further study:

Question 8.2 Can one refine the naive approach discussed above in order to exhibit embedded "exotic" matching paths and Lagrangian spheres in $X_{i}$ ?

If not, what is a good way to algebraize the distinction between "embedded" and "immersed" relations among Dehn twists in a mapping class group factorization?

\section{References}

[1] D Auroux, Fiber sums of genus 2 Lefschetz fibrations, Turkish J. Math. 27 (2003) 1-10 MR1975329

[2] D Auroux, S K Donaldson, L Katzarkov, Luttinger surgery along Lagrangian tori and non-isotopy for singular symplectic plane curves, Math. Ann. 326 (2003) 185-203 MR1981618

[3] D Auroux, L Katzarkov, A degree doubling formula for braid monodromies and Lefschetz pencils, preprint

[4] D Auroux, L Katzarkov, Branched coverings of $\mathbf{C P}^{2}$ and invariants of symplectic 4-manifolds, Invent. Math. 142 (2000) 631-673 MR1804164

[5] D Auroux, V Muñoz, F Presas, Lagrangian submanifolds and Lefschetz pencils, J. Symplectic Geom. 3 (2005) 171-219 MR2199539

[6] D Auroux, I Smith, Lefschetz pencils, branched covers and symplectic invariants, from: "Proc. CIME school, Symplectic 4-manifolds and algebraic surfaces (Cetraro, 2003)", Lecture Notes in Math., Springer , to appear

[7] S K Donaldson, Lefschetz fibrations in symplectic geometry, from: "Proceedings of the International Congress of Mathematicians, Vol. II (Berlin, 1998)", Extra Vol. II (1998) 309-314 (electronic) MR1648081

[8] S K Donaldson, Lefschetz pencils on symplectic manifolds, J. Differential Geom. 53 (1999) 205-236 MR1802722

[9] R Fintushel, R J Stern, Symplectic surfaces in a fixed homology class, J. Differential Geom. 52 (1999) 203-222 MR1758295 
[10] R Friedman, J W Morgan, Algebraic surfaces and Seiberg-Witten invariants, J. Algebraic Geom. 6 (1997) 445-479 MR1487223

[11] T Fuller, Diffeomorphism types of genus 2 Lefschetz fibrations, Math. Ann. 311 (1998) 163-176 MR1624287

[12] R E Gompf, Toward a topological characterization of symplectic manifolds, J. Symplectic Geom. 2 (2004) 177-206 MR2108373

[13] R E Gompf, A I Stipsicz, 4-manifolds and Kirby calculus, Graduate Studies in Mathematics 20, Amer. Math. Soc. (1999) MR1707327

[14] M Gromov, Pseudoholomorphic curves in symplectic manifolds, Invent. Math. 82 (1985) 307-347 MR809718

[15] H Hofer, V Lizan, J-C Sikorav, On genericity for holomorphic curves in fourdimensional almost-complex manifolds, J. Geom. Anal. 7 (1997) 149-159 MR1630789

[16] E Horikawa, Algebraic surfaces of general type with small $C_{1}^{2}$. I, Ann. of Math. (2) 104 (1976) 357-387 MR0424831

[17] A Kas, On the handlebody decomposition associated to a Lefschetz fibration, Pacific J. Math. 89 (1980) 89-104 MR596919

[18] D McDuff, Almost complex structures on $S^{2} \times S^{2}$, Duke Math. J. 101 (2000) 135-177 MR1733733

[19] D McDuff, D Salamon, $J$-holomorphic curves and symplectic topology, American Mathematical Society Colloquium Publications 52, American Mathematical Society, Providence, RI (2004) MR2045629

[20] MJ Micallef, B White, The structure of branch points in minimal surfaces and in pseudoholomorphic curves, Ann. of Math. (2) 141 (1995) 35-85 MR1314031

[21] B G Moishezon, Stable branch curves and braid monodromies, from: "Algebraic geometry (Chicago, Ill., 1980)", Lecture Notes in Math. 862, Springer, Berlin (1981) 107-192 MR644819

[22] P Seidel, More about vanishing cycles and mutation, from: "Symplectic geometry and mirror symmetry (Seoul, 2000)”, World Sci. Publ., River Edge, NJ (2001) 429-465 MR1882336

[23] B Siebert, G Tian, On hyperelliptic $C^{\infty}$-Lefschetz fibrations of four-manifolds, Commun. Contemp. Math. 1 (1999) 255-280 MR1696101

[24] B Siebert, G Tian, On the holomorphicity of genus two Lefschetz fibrations, Ann. of Math. (2) 161 (2005) 959-1020 MR2153404

[25] J-C Sikorav, The gluing construction for normally generic $J$-holomorphic curves, from: "Symplectic and contact topology: interactions and perspectives (Toronto, ON/Montreal, QC, 2001)", Fields Inst. Commun. 35, Amer. Math. Soc., Providence, RI (2003) 175-199 MR1969276

Geometry ${ }^{6}$ Topology, Volume 10 (2006) 
[26] I Smith, Lefschetz pencils and divisors in moduli space, Geom. Topol. 5 (2001) 579-608 MR1833754

Department of Mathematics, MIT, Cambridge, MA 02139, USA

auroux@math.mit.edu

Proposed: Rob Kirby

Received: 28 May 2006

Seconded: Ronald Fintushel, Ronald Stern

Revised: 25 September 2006

Geometry 83 Topology, Volume 10 (2006) 